\title{
The Role of the Internal Energy in MMCs Operating in Grid-Forming Mode
}

\author{
Enric Sánchez-Sánchez, Student Member, IEEE, Eduardo Prieto-Araujo, Member, IEEE, \\ and Oriol Gomis-Bellmunt, Senior Member, IEEE
}

\begin{abstract}
With an increasing number of renewable energy resources being integrated into the power system through power electronics, conventional power plants based on large synchronous machines tend to be reduced in the upcoming future. With the Modular Multilevel Converter as the state-of-the-art power electronics solution for power systems, it will be necessary to operate some of these converters in grid-forming mode, in order to either preserve the stability and robustness of the ac grid or as the only solution to generate the ac grid in cases with no available synchronous generation. This paper aims to understand how the internal energy control of the MMC can be used to effectively mitigate the propagation of ac power disturbances to the dc side, thanks to its buffering capability. Different energy control structures are explored, and a comprehensive discussion and comparison among them is provided, including stability analysis, transient performance and tuning guidelines. Simplified and linear models are used to address the analysis, whereas detailed non-linear models implemented in MATLAB Simulink are used to verify the results via time-domain simulations.
\end{abstract}

Index Terms-MMC, energy control, grid-forming, tuning, interaction mitigation, HVDC.

\section{INTRODUCTION}

$\mathbf{I}$ $\mathrm{N}$ the upcoming future of power systems, conventional synchronous generation will experience a progressive reduction, to the benefit of renewable energy generation, such as wind and solar, in order to meet the environmental goals planned for the future [1]. This implies that new power electronics converters will be installed in order to integrate these renewable resources, to the detriment of the large mechanical rotating machines from classic thermal power plants. With such a reduction of rotating mass, some of the aforementioned converters will be responsible of handling the stability and the robustness of the power system by means of operating in gridforming mode.

Grid-forming mode of operation is a well-known concept, and several studies for microgrids [2]-[6] and power system applications [7]-[9] have been addressed in the literature in the recent years. The two main grid-forming approaches are the Virtual Synchronous Machine (VSM) approach [3] and the frequency-droop approach [10]. In fact, it is shown

This work was partially funded by FEDER / Ministerio de Ciencia, Innovación y Universidades - Agencia Estatal de Investigación, Project RTI2018095429-B-I00, and by the FI-AGAUR Research Fellowship Program, Generalitat de Catalunya.

E. Sánchez-Sánchez, E. Prieto-Araujo, and O. Gomis-Bellmunt are with the Centre d'Innovació Tecnològica en Convertidors Estàtics Accionaments, Departament d'Enginyeria Elèctrica, Universitat Politècnica de Catalunya, Barcelona 08028, Spain. E. Prieto-Araujo is also a Serra Húnter Lecturer (e-mail: enric.sanchez.sanchez@citcea.upc.edu; eduardo.prietoaraujo@citcea.upc.edu; oriol.gomis@upc.edu) in [11] that both control approaches are equivalent. Most of these studies deal with 2-level Voltage Source Converters (2L-VSCs), focusing on the ac side, whereas only a handful of references are found with respect to grid-forming mode for power system applications using MMCs, as summarized next. Two different controllers in VSC-HVDC interconnectors operating in parallel are compared in [12], and an enhanced control for an offshore wind farm station is discussed in [13]. Despite using MMC models in both articles, they focus on the ac voltage and frequency control, with no discussion about the internal energy control. In [14], a dynamic protection integrated model predictive control concept is developed and applied to the MMC for grid restoration purposes, focusing on avoiding MMC overcurrents. An ac fault analysis of islanded grid including MMCs operating in grid-forming mode is presented in [15], also focusing on the ac side control structure. In this case, instead of using an energy-based approach, a circulating current suppressing control (CCSC) scheme is used, meaning that the energy is not explicitly controlled. In [16], a classic grid-forming control scheme together with a full energy-based control is implemented in an MMC and successfully tested in laboratory, discussing the implications of having a storage system in the dc side or inside the MMC submodules. Furthermore, the VSM concept has been recently extrapolated and implemented in the MMC [17], [18].

The buffering capabilities that the MMC offers due to its topology, i.e., due to the internal energy stored in its submodules capacitors, has been a subject of interest within the scientific community over the last years. First of all, the modulation strategy plays a relevant role in the effectiveness of this feature, which in general can be implemented in two ways. On one hand, Uncompensated Modulation (UCM) does not include any compensation for the impact of the oscillations in the equivalent arm capacitance voltages on the modulated output voltages, i.e., the modulation indexes are calculated using the dc voltage. On the other hand, Compensated Modulation (CM) includes an online compensation for those oscillations, i.e., the modulation indexes are calculated using the measured arm voltages. As an example, regarding grid-following MMCs operating in PQ mode, in [19] it is shown how the approach from [20] (UCM) fails to withstand an unbalanced voltage sag, distorting the dc side. Results are notably improved in the case of [21] (CM), and further improved when inner circulating current control loops are included [19].

Regarding unbalanced operation, in [22], a control approach aiming to reduce dc side power oscillations is presented, filtering the $100 \mathrm{~Hz}$ component out of the zero-sequence 
additive current, showing the firewall capability of the MMC. A more elaborated approach from the same authors [23] deals with optimal circulating currents reference calculation directly in the $a b c$ frame, that effectively decouples the ac-grid conditions from the dc bus, not only in steady-state but also in transient unbalanced conditions in a grid-following MMC, improving and extending results from [24].

Other works deal with control aspects of grid-following MMCs operating in dc voltage control mode. In that case, with a disturbance in the dc grid, a different transient performance in the dc and ac sides is achieved depending on the control structure adopted. A virtual capacitor strategy is proposed in [25], which is able to boost the effective capacitance of the dc side and improve the dc dynamics. In [26], [27] it is shown that interchanging the ac and dc current control references in an MMC controlling the dc voltage allows for decoupling the ac and dc sides, avoiding interactions. It can be noted that in terms of the MMC physical limitations, the control design criteria for grid-forming and grid-following converters can be similar, as these objectives would typically focus in reducing overshoots, avoiding undesired oscillations or obtaining a specific settling time. The fundamental difference, however, is that in grid-following mode the AC power is a control input, whereas in grid-forming mode it is a physical disturbance.

In this context, there is gap in the literature in terms of analyzing potential capabilities that the MMC offers in grid-forming mode, for instance in offshore $\mathrm{AC}$ wind farm or passive islanded systems. This paper aims to analyze and provide a comprehensive discussion about the features of this buffering capability in such scenarios. The AC side grid-forming control used in the present work is equivalent to the classic frequency droop used in $2 \mathrm{~L}-\mathrm{VSCs}$, and the comparison or proposal of new grid-forming controls is not the matter of the present study. However, the extra degree of freedom due to the total internal energy of the MMC can play a relevant role in the dynamic performance of the system. The contribution of this paper is to analyze different energy control structures, highlighting their differences in transient conditions, and providing control design guidelines to obtain the desired performance. Different case scenarios with one and multiple grid-forming converters are simulated using MATLAB Simscape Power Systems, in order to validate the analysis and to highlight the superiority of some of the structures.

\section{SYSTEM DESCRIPTION}

The present study deals with MMCs operating in gridforming mode. The converter is connected to an HVDC network in the dc side, which provides a stable dc voltage, typically controlled by one or more converters of the HVDC network. On the ac side, the converter controls the amplitude, frequency and angle of the voltage at the point of connection, acting as a slack bus. A list of examples where grid-forming converters can be used at the transmission system level are described below.

- Offshore AC wind farm clusters, where one or more converters impose the ac grid voltage to export the power generated in the offshore wind farm, which is typically exported to the mainland grid through HVDC (Fig. 1a).

- Passive islanded systems, i.e., islands where the main part or all of the generation needs to be imported from outside, e.g., through one or more HVDC links (Fig. 1b).

- Weak grids, i.e., grids with a low short-circuit ratio that need one or more grid-forming converters to stabilize the grid, or to make it robust.

In the case of islanded systems, if there is enough synchronous generation, the converter/s that import or export power (GF 1 and GF 2, Fig. 1) do not necessarily have to operate in grid-forming mode, and might operate in gridfollowing mode instead if the ac grid is strong enough. Equivalently, this can be also the case of weak grids. In those cases, the converter can optionally operate in grid-forming mode to support the existing ac grid. However, in other cases such as offshore ac wind farms (Fig. 1a) and islanded systems with no synchronous generation (Fig. 1b), it is in general necessary that one or more converters operate in grid-forming mode, as there is no ac grid available. As compared to gridfollowing, grid-forming mode allows the converter to remain in operation after a large or full trip in the ac side, and it can also provide black-start capability. It has to be noted that the ac power acts as a disturbance to the system, instead of as a control input.

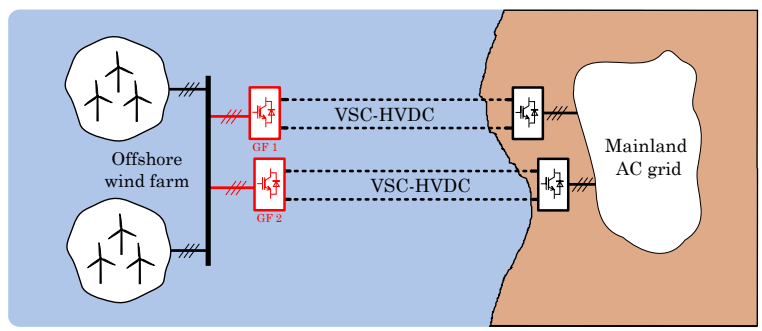

(a) Offshore wind power plant connected to land via HVDC

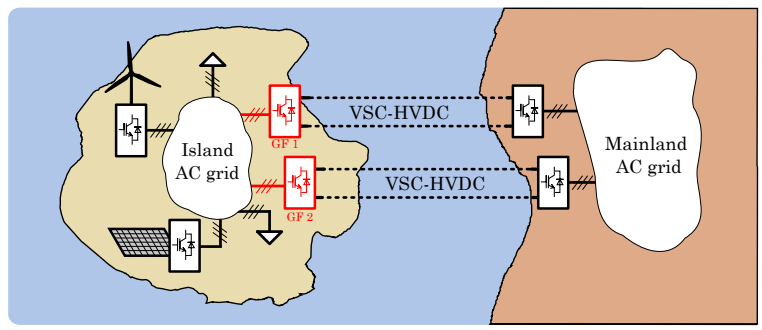

(b) Islanded system connected to main land grid via HVDC

Fig. 1. Examples of applications of grid-forming MMCs.

\section{SYSTEM MODELLING}

\section{A. MMC electrical model}

The MMC topology shown in Fig. 2 consists of six arms, each of them including $N_{\text {arm }}$ half-bridge submodules with a capacitance $C_{\mathrm{SM}}$, and an arm reactor in series. The submodules are controlled individually, either inserting or bypassing the capacitor. The three legs correspond to the three phases ( $a$, $b$ and $c$ ), each containing two arms. The six arms synthesize the required voltages in order to achieve the desired power 
exchange between the $\mathrm{AC}$ and the dc side and to handle the internal energy balance of the converter.

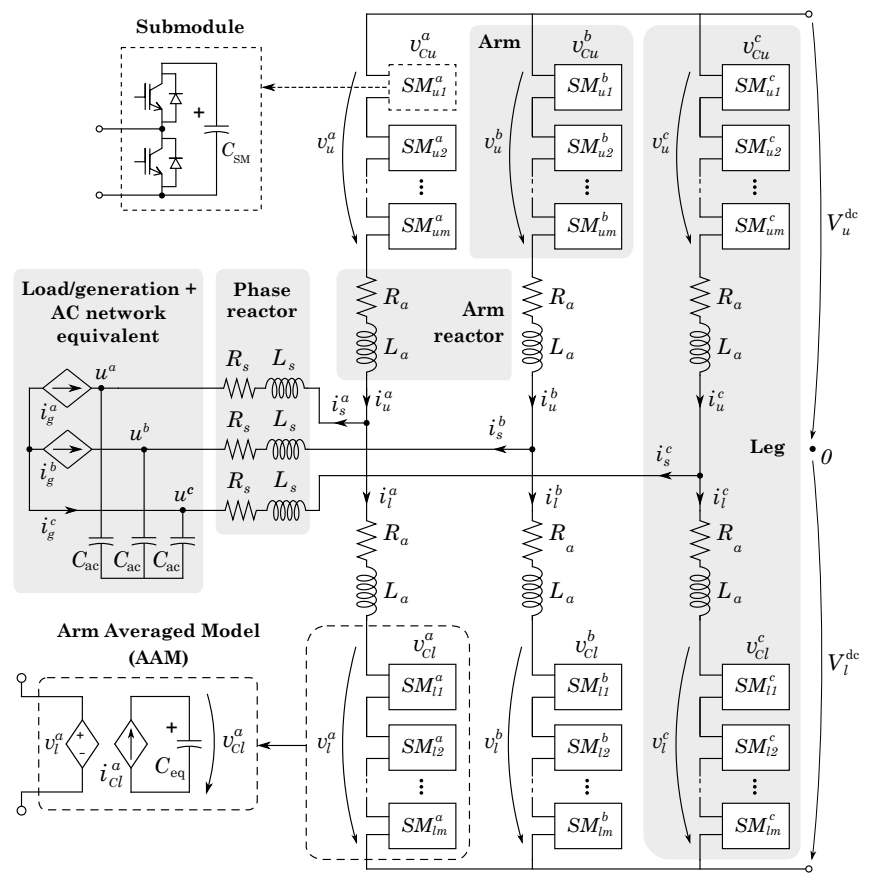

Fig. 2. Electrical model of MMC connected to an AC network (including lines or cables) with load or generation.

A coordinates transformation from the upper - lower to a diff-sum reference frame is typically used [21], as it facilitates the state-space representation of the MMC and also allows for the control of the different degrees of freedom of the converter. Hence, following the same conventions as in [19], [27], the following variable change is defined

$$
\left\{\begin{array}{l}
v_{\mathrm{diff}}^{j} \triangleq \frac{1}{2}\left(-v_{u}^{j}+v_{l}^{j}\right) \\
v_{\mathrm{sum}}^{j} \triangleq v_{u}^{j}+v_{l}^{j} \\
i_{\mathrm{sum}}^{j} \triangleq \frac{1}{2}\left(i_{u}^{j}+i_{l}^{j}\right)
\end{array}, \quad\left\{\begin{array}{l}
R_{\mathrm{eq}} \triangleq R_{s}+\frac{R_{a}}{2} \\
L_{\mathrm{eq}} \triangleq L_{s}+\frac{L_{a}}{2}
\end{array}\right.\right.
$$

with

$v_{\text {diff }}^{j}$ : differential voltage (middle point of the arm),

$v_{\text {sum }}^{j}$ : additive voltage (approx. equal to the dc voltage),

$i_{\text {sum }}^{j}$ : additive current (from upper to lower arm),

$R_{a}, L_{a}:$ arm resistance and inductance,

$R_{s}, L_{s}$ : transformer equivalent resistance and inductance,

$C_{\mathrm{ac}}$ : equivalent ac capacitance in the point of connection.

Assuming a balanced dc side, a balanced and grounded ac neutral point, and using the change of variables in (1) the ac side and circulating current equations are

$$
\begin{gathered}
v_{\mathrm{diff}}^{a b c}-u^{a b c}=R \mathcal{I}_{\mathbf{3}} i_{s}^{a b c}+L \mathcal{I}_{\mathbf{3}} \frac{\mathrm{d} i_{s}^{a b c}}{\mathrm{~d} t} \\
i_{s}^{a b c}-i_{g}^{a b c}=C_{\mathrm{ac}} \mathcal{I}_{\mathbf{3}} \frac{\mathrm{d} u^{a b c}}{\mathrm{~d} t} \\
v_{\mathrm{sum}}^{a b c}-V_{t}^{\mathrm{dc}}\left(\begin{array}{lll}
1 & 1 & 1
\end{array}\right)^{T}=-2 R_{a} \mathcal{I}_{\mathbf{3}} i_{\mathrm{sum}}^{a b c}-2 L_{a} \mathcal{I}_{\mathbf{3}} \frac{\mathrm{d} i_{\mathrm{sum}}^{a b c}}{\mathrm{~d} t},
\end{gathered}
$$

where $u^{a b c}$ denotes the PCC voltage, $i_{s}^{a b c}$ is the ac grid current, i.e., $i_{s}^{j}=i_{u}^{j}-i_{l}^{j}$ for $j \in\{a, b, c\}, V_{t}^{\mathrm{dc}}$ is the dc side voltage, and $\mathcal{I}_{\mathbf{n}}$ refers to a $n \times n$ identity matrix. The voltages and currents in the arms can contain an ac and a dc component, with different roles in the power exchange and the energy balance inside the converter (see [19] for a thorough analysis). The Average Arm Model (AAM) is adopted (Fig. 2), which assumes that each individual submodule capacitor voltage is close to its nominal value, thus allowing for a representation of the whole arm by an equivalent capacitor. This model and variations of the same concept are well established in the recent literature [28], [29], as well as experimental valiadation [30]. The equivalent capacitor voltage of each arm $v_{C u l}^{a b c}$ depends on the power exchanged by the arm, which is reflected as a current $i_{C u l}^{a b c}$ in each capacitor circuit:

$$
i_{C u l}^{a b c}=C_{\mathrm{eq}} \mathcal{I}_{\mathbf{6}} \frac{\mathrm{d} v_{C u l}^{a b c}}{\mathrm{~d} t}
$$

\section{B. MMC grid-forming control}

A classic grid-forming control for VSCs consists of an outer ac voltage vector control plus an inner ac current vector control $\left(u-i_{s}\right)$, both using PI controllers in $q d 0^{*}$ frame. Additionally, a frequency droop using the active power is included to achieve active power sharing in a multiple converter setup. In addition, an optional ac voltage reference droop using the reactive power might be also included, which can be useful to achieve reactive power sharing. This scheme is identical for both $2 \mathrm{~L}-\mathrm{VSC}$ and MMC-VSCs [15].

In the case of MMCs, additional loops to control the internal energy balance might be added. Purposely, in the present study, an energy-based approach is used, meaning that the internal energy of the MMC is explicitly controlled via closed-loop control. The overall energy control can be seen as one total energy PI controller in addition to five extra PI controllers that deal with the balancing among legs (horizontal balancing) and the individual leg upper-lower balancing (vertical balancing). Also, three inner additive current loops are usually included (see Fig. 3). It is important to note that typically the output filter of an MMC would not include a capacitor, being composed only by an RL equivalent related to the coupling transformer. Therefore, the effective capacitance depends on the topology of the ac grid that the MMC is connected to. This implies that the current $i_{g}$ (optionally used as a feed-forward signal in the ac voltage control in 2L-VSCs) cannot be directly measured.

A more detailed control scheme is shown in Fig. 4, where the horizontal and vertical balancing control and the corresponding additive current controllers have not been included for simplicity. Also, note that a low-pass filter is usually included for the active and reactive power measurements used in the corresponding droop schemes [8].

\section{Small-signal model of the system}

A linearized small-signal model of the system is derived to enable linear analysis and linear control design. The equations

\footnotetext{
* In the present work, electrical machines notation from [31] is adopted, meaning that $d$ and $q$ components are interchanged, instead of using the more common $d q 0$ notation.
} 


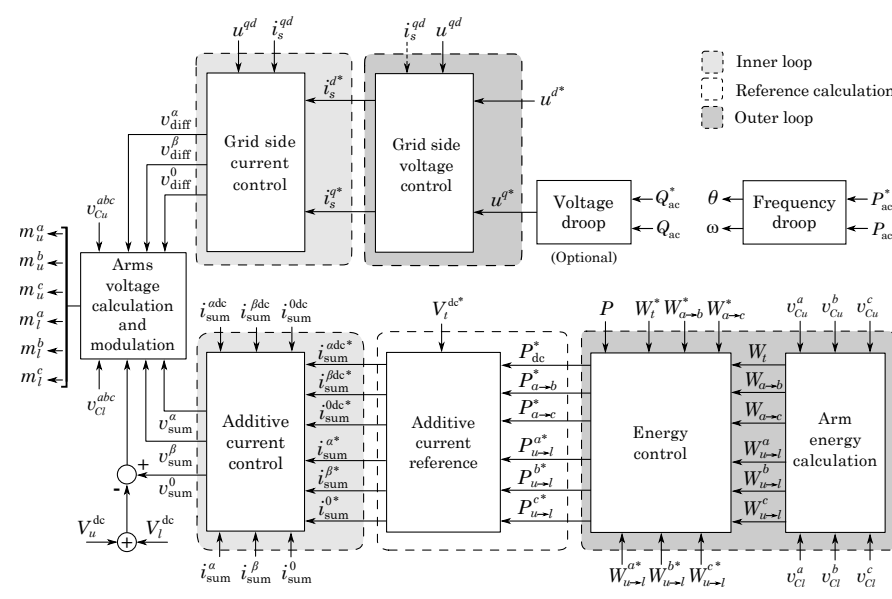

Fig. 3. MMC overall control system operating as grid-forming.

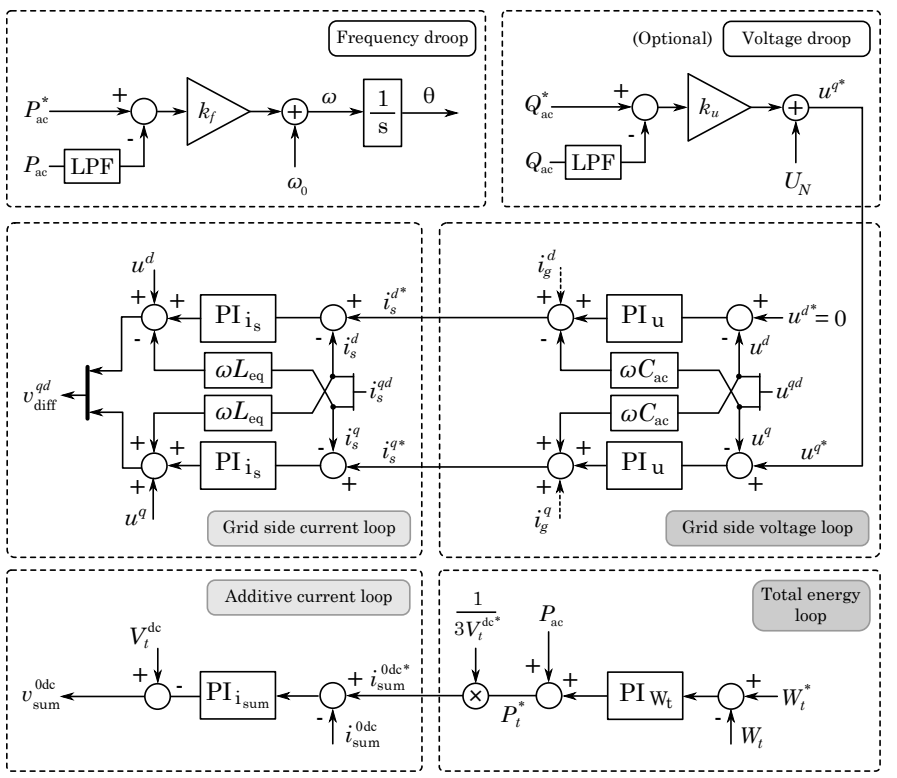

Fig. 4. Detailed MMC grid-forming control, without showing vertical and horizontal balancing control for simplicity.

of each subsystem and their linearization, if necessary, are detailed next.

1) MMC ac side and ac grid dynamics: The converter ac grid coupling filter (i.e., RL transformer equivalent) (6)(9) and the ac network (in this case, single $\pi$-section cable equivalent) (10)-(13) are equivalent to the $2 \mathrm{~L}-\mathrm{VSC}$ case, with the difference that in (6) and (7) $R_{\text {eq }}$ and $L_{\text {eq }}$ include the arm filter parameters (see (1)). The electrical circuit is shown in Fig. 5. As a frequency droop strategy is used, (6)-(13) are linearized with respect to the frequency to account for the frequency variation effect. The ac capacitor $C_{\mathrm{ac}}$ is an equivalent capacitance that includes the cables related capacitance and a physical filter capacitor if desired, although this is normally not included in MMCs. Subindex $c$ refers to the cable $\left(R_{c}\right.$, $L_{c}$ and $C_{c}$ are the cable parameters), $g$ refers to the ac grid ( $i_{g}$ and $u_{g}$ are the current through the cable and the voltage at the load end of the cable), and $l$ refers to the load (being $i_{l}$ the load current). Note that in the present case $C_{\mathrm{ac}}=C_{c} / 2$.

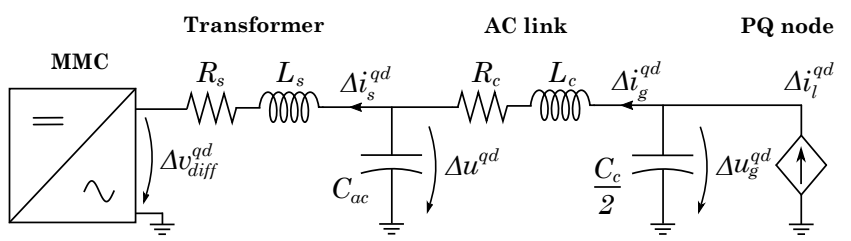

Fig. 5. Electrical circuit of the MMC ac side, the ac cable and the PQ node.

$$
\begin{gathered}
\Delta \dot{i_{s}^{q}}=-\frac{R_{\mathrm{eq}}}{L_{\mathrm{eq}}} \Delta i_{s}^{q}-i_{s 0}^{d} \Delta \omega-\omega_{0} \Delta i_{s}^{d}+\frac{1}{L_{\mathrm{eq}}} \Delta v_{\mathrm{diff}}^{q}-\frac{1}{L_{\mathrm{eq}}} \Delta u^{q} \\
\Delta \dot{i}_{s}^{d}=-\frac{R_{\mathrm{eq}}}{L_{\mathrm{eq}}} \Delta i_{s}^{d}+i_{s 0}^{q} \Delta \omega-\omega_{0} \Delta i_{s}^{q}+\frac{1}{L_{\mathrm{eq}}} \Delta v_{\mathrm{diff}}^{d}-\frac{1}{L_{\mathrm{eq}}} \Delta u^{d} \\
\Delta \dot{u}^{q}=-\frac{1}{C_{\mathrm{ac}}} \Delta i_{s}^{q}-u_{0}^{d} \Delta \omega-\omega_{0} \Delta u^{d}-\frac{1}{C_{\mathrm{ac}}} \Delta i_{g}^{q} \\
\Delta \dot{u}^{d}=-\frac{1}{C_{\mathrm{ac}}} \Delta i_{s}^{d}+u_{0}^{q} \Delta \omega-\omega_{0} \Delta u^{q}-\frac{1}{C_{\mathrm{ac}}} \Delta i_{g}^{d} \\
\Delta i_{g}^{q}=-\frac{R_{c}}{L_{c}} \Delta i_{g}^{q}-i_{g 0}^{q} \Delta \omega-\omega_{0} \Delta i_{g}^{d}+\frac{1}{L_{c}} \Delta u_{g}^{q}-\frac{1}{L_{c}} \Delta u_{g}^{q} \\
\Delta \dot{i}_{g}^{d}=-\frac{R_{c}}{L_{c}} \Delta i_{g}^{d}+i_{g 0}^{d} \Delta \omega-\omega_{0} \Delta i_{g}^{q}+\frac{1}{L_{c}} \Delta u_{g}^{d}-\frac{1}{L_{c}} \Delta u_{g}^{d} \\
\Delta \dot{u}_{g}^{q}=-\frac{2}{C_{c}} \Delta i_{g}^{q}-u_{g 0}^{d} \Delta \omega-\omega_{0} \Delta u_{g}^{d}-\frac{2}{C_{c}} \Delta i_{l}^{q} \\
\Delta \dot{u}_{g}^{d}=-\frac{2}{C_{c}} \Delta i_{g}^{d}+u_{g 0}^{q} \Delta \omega-\omega_{0} \Delta u_{g}^{q}-\frac{2}{C_{\mathrm{c}}} \Delta i_{l}^{d}
\end{gathered}
$$

2) MMC internal dynamics and control: As exhaustively discussed in [32], [33], including the overall internal energy dynamics requires an extensive mathematical manipulation. Thereby, a simplified model that considers only the total energy dynamics of the MMC [34] will be used in the present study (14). Assuming that the horizontal and vertical energy balancing controllers are properly tuned, that model effectively captures the overall ac/dc dynamics. Also, an extra equation for the additive current of the MMC is required (15):

$$
\begin{aligned}
\Delta \dot{W}_{t}= & -\frac{3}{2}\left(\Delta v_{\mathrm{diff}}^{q} i_{s 0}^{q}+\Delta v_{\mathrm{diff}}^{d} i_{s 0}^{d}+\Delta i_{s}^{q} v_{\mathrm{diff} 0}^{q}+\Delta i_{s}^{d} v_{\mathrm{diff} 0}^{d}\right) \\
& +3\left(\Delta i_{\mathrm{sum}}^{\mathrm{Odc}} v_{\mathrm{sum} 0}^{0 \mathrm{dc}}+\Delta v_{\mathrm{sum}}^{\mathrm{ddc}} i_{\mathrm{sum} 0}^{\mathrm{Odc}}\right) \\
\Delta \dot{i}_{\mathrm{sum}}^{\mathrm{ddc}}= & -\frac{R_{a}}{L_{a}} \Delta i_{\mathrm{sum}}^{\mathrm{Odc}}-\frac{1}{2 L_{a}} \Delta v_{\mathrm{sum}}^{0 \mathrm{dc}}+\frac{1}{2 L_{a}} \Delta V_{t}^{\mathrm{dc}}
\end{aligned}
$$

Moreover, active and reactive power signals, which are necessary for some of the control loops, are linearized as

$$
\begin{gathered}
\Delta P_{\mathrm{ac}}=\frac{3}{2}\left(\Delta u^{q} i_{s 0}^{q}+\Delta u^{d} i_{s 0}^{d}+\Delta i_{s}^{q} u_{0}^{q}+\Delta i_{s}^{d} u_{0}^{d}\right) \\
\Delta Q_{\mathrm{ac}}=\frac{3}{2}\left(-\Delta u^{q} i_{s 0}^{q}+\Delta u^{d} i_{s 0}^{d}+\Delta i_{s}^{q} u_{0}^{q}-\Delta i_{s}^{d} u_{0}^{d}\right)
\end{gathered}
$$

The equations related to the current control, voltage control, energy control, frequency droop and voltage droop are not included in the paper for simplicity, as they can be readily derived from the control scheme (Fig. 4). A block diagram of the overall linear model is shown in Fig. 6. 

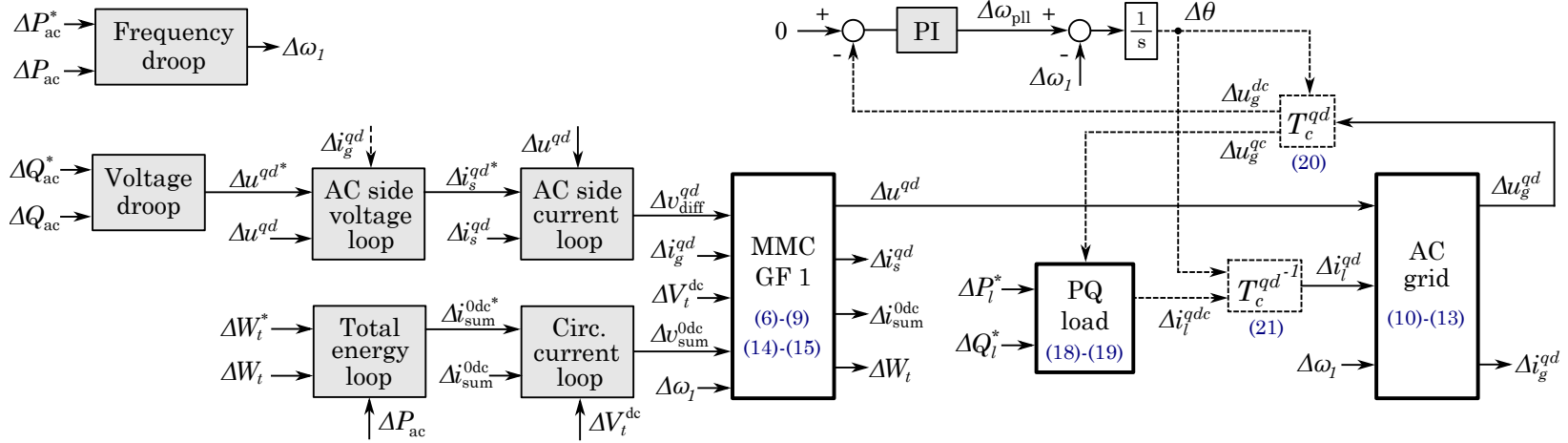

Fig. 6. Linear model of an ac grid with one grid-forming MMC and one PQ nodes.

3) Disturbance dynamics: A constant power node is considered at the other end of the ac cable, injecting or absorbing $\mathrm{P}$ and/or Q through controllable current sources. This corresponds to the offshore wind generators from Fig. 1a (power flowing towards the grid-forming MMC), which typically use power electronics converters synchronized with the offshore ac grid through Phase-Locked Loops (PLL). On the other hand, a scenario such as an islanded system (Fig. 1b) would typically involve different types of loads (constant current, constant impedance and constant power loads), making its dynamic model more complex. Therefore, for convenience in the analysis, the case of a constant power node representing a power electronics interface with a PLL will be considered.

Instead of modelling a detailed converter, the power references $\Delta P_{l}^{*}(s)$ and $\Delta Q_{l}^{*}(s)$ are filtered using first-order transfer functions that emulate the ac power closed-loop response of the converter, with a time constant of $\tau_{p}$. A PLL [35] tracks the voltage angle by means of controlling its $d$ component to zero. Then, according to instantaneous power theory [36], the $q d$ components of the current are calculated as (18)-(19). An extra first-order filter (time constant of $\tau_{c}$ ) is included, representing the dynamics of the current loops $\left(\tau_{c}\right.$ is typically around 10 times lower than $\tau_{p}$ ).

$$
\begin{aligned}
& \Delta i_{l}^{q}(s)=\frac{2}{3}\left(\frac{1}{u_{g 0}^{q}} \frac{\Delta P_{l}^{*}(s)}{\tau_{p} s+1}-\frac{P_{l 0}}{u_{g 0}^{q}{ }^{2}} \Delta u_{g}^{q}(s)\right) \frac{1}{\tau_{c} s+1} \\
& \Delta i_{l}^{d}(s)=\frac{2}{3}\left(\frac{1}{u_{g 0}^{q}} \frac{\Delta Q_{l}^{*}(s)}{\tau_{p} s+1}-\frac{Q_{l 0}}{u_{g 0}^{q}{ }^{2}} \Delta u_{g}^{q}(s)\right) \frac{1}{\tau_{c} s+1}
\end{aligned}
$$

In order to integrate the different elements into the linear model, a common $q d$ reference frame has to be established. In this case, the reference angle will be the one generated by the grid-forming MMC. The PLL is modeled as a closed-loop with a PI controller that regulates the $d$ component of the measured voltage to zero, as mentioned before. The output of the PI, i.e., the PLL frequency $\Delta \omega_{\text {pll }}$, minus the reference frequency $\Delta \omega_{1}$ is integrated to obtain $\Delta \Theta$, which is the angle difference between the PQ node and the grid-forming MMC angles. This $\Delta \Theta$ is necessary to transform any $q d$ magnitude from one reference to another. In this case, PQ node magnitudes $(q d c)$ are transformed to the common reference $(q d)$ using (20), and vice-versa using (21) [37] (see Appendix A for the detailed transformation matrices).

$$
\begin{gathered}
\Delta x^{q d c}=\mathbf{T}_{\mathbf{c}}^{\mathbf{q d}}\left(\Delta x^{q d}, \Delta e_{\theta}\right)^{T} \\
\Delta x^{q d}=\mathbf{T}_{\mathbf{c}}^{\mathbf{q d}^{-1}}\left(\Delta x^{q d c}, \Delta e_{\theta}\right)^{T}
\end{gathered}
$$

\section{TOTAL ENERGY CONTROL STRUCTURES}

In $2 \mathrm{~L}-\mathrm{VSCs}$, the ac and dc powers are fully coupled, meaning that any disturbance in the ac side will propagate to the dc side, and vice versa. In MMCs, on the contrary, the internal total energy dynamics will have a determining impact on how the ac/dc interaction and disturbance propagation occurs, offering a buffering capability that can be used as desired, depending on the specifications of the converter and its application. When an ac power disturbance occurs, a portion of that power will charge or discharge the MMC internal arm capacitors, and another portion will flow to or from the dc side (Fig. 7). The way this power is transiently distributed will depend on what control structure is used and how the tunable control parameters are set.

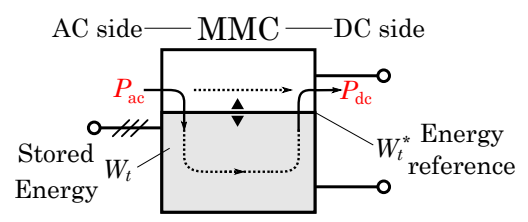

Fig. 7. Conceptual representation of how an ac disturbance is partly absorbed by the MMC and partly sent to the dc side.

As compared to an MMC controlling the dc voltage, where several control structure combinations might make sense [27], the structure of the grid-forming MMC control appears to be more evident. On one hand, the classic frequency droop control including a cascaded ac voltage and current vector control used in $2 \mathrm{~L}-\mathrm{VSCs}$ is directly applicable to the MMC, without any difference. On the other hand, it seems to be obvious that the energy controller has to be, therefore, cascaded with the inner additive current, which is the other part of the MMC control. Nevertheless, there are still different alternatives in terms of how to implement the energy control, that will affect the internal MMC and the dc dynamics in different ways. 
In order to analyze this mechanism in a comprehensive way, the following assumptions are recalled:

- The dc voltage is constant.

- The zero component circulating current closed loop (i.e., dc component) is simplified to a first-order transfer function with time constant $\tau_{\text {sum }}$ (faster than the outer loops).

- The ac power is a disturbance of the system.

The dynamics of the dc power and the MMC internal total energy are defined, respectively, in the Laplace domain as

$$
\begin{aligned}
& P_{\mathrm{dc}}(s)=V_{\mathrm{dc}} I_{\mathrm{dc}}(s)=V_{\mathrm{dc}}\left(3 \frac{I_{\mathrm{sum}}^{0 \mathrm{dc}^{*}}(s)}{\tau_{\mathrm{sum}} s+1}\right)=\frac{P_{\mathrm{dc}}^{*}(s)}{\tau_{\mathrm{sum}} s+1} \\
& s W_{t}(s)=\frac{1}{s}\left(P_{\mathrm{dc}}(s)-P_{\mathrm{ac}}(s)\right)=\frac{P_{\mathrm{dc}}^{*}(s)}{\tau_{\mathrm{sum}} s+1}-P_{\mathrm{ac}}(s)
\end{aligned}
$$

Both (22) and (23) depend on the expression of $P_{\mathrm{dc}}^{*}(s)$, which depends on the control structure adopted, as will be seen next. Also, and for compactness later on, the control action of the total energy PI controller is defined as

$$
G_{c}(s)=k_{p}\left(W_{t}^{*}(s)-W_{t}(s)\right)+\frac{k_{i}}{s}\left(W_{t}^{*}(s)-W_{t}(s)\right)
$$

\section{A. Coupled control approach}

A total energy controller with a feed-forward of the active power (Fig. 4) is a typical structure used in MMCs operating in PQ mode [19], [38]-[40], and also in de voltage control mode [27], [41]. Hereinafter, this structure will be referred to as coupled energy control. This structure is somehow a logical extrapolation from the 2L-VSC control, where the ac and dc powers are instantly balanced, as they are physically linked. In the MMC case, using the ac power measurement as a feed-forward signal forces a coupling between both sides. Therefore, it is expected that the dynamics of the MMC under this control scheme will be remarkably similar than those of the 2L-VSC. As will be seen next, the flexibility that this structure offers is highly limited. From (25) it is observed that $P_{\mathrm{dc}}(s)$ will react fast to the disturbance $P_{\mathrm{ac}}(s)$. The only limitation to propagate the disturbance to the dc side depends on $\tau_{\text {sum }}$, which is relatively small, i.e., the transient is fast. Then, as seen in the first term of the right-hand side of (26), the energy is not highly disturbed by $P_{\mathrm{ac}}(s)$, as its coefficient is close to zero. The resulting small deviation of the energy, therefore, will be compensated by the PI controller, which will have a small effect in $P_{\mathrm{dc}}(s)$.

$$
\begin{gathered}
P_{\mathrm{dc}}(s)=\frac{P_{\mathrm{ac}}(s)}{\tau_{\mathrm{sum}} s+1}+\frac{G_{c}(s)}{\tau_{\mathrm{sum}} s+1} \\
s W_{t}(s)=\left(\frac{P_{\mathrm{ac}}(s)}{\tau_{\mathrm{sum}} s+1}-P_{\mathrm{ac}}(s)\right)+\frac{G_{c}(s)}{\tau_{\mathrm{sum}} s+1}
\end{gathered}
$$

\section{B. Power-filtered control approach}

Keeping the previous control structure, a low-pass filter (LPF, time constant $\left.\tau_{g 2}\right)$ applied to $P_{\mathrm{ac}}(s)$ is proposed. The delay caused by this filter will force the internal energy of the MMC to smoothly increase, thus acting as a buffer between the

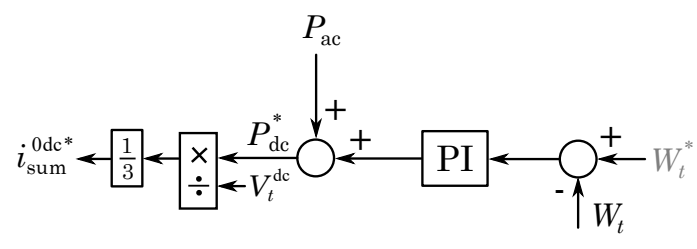

Fig. 8. Coupled energy control approach.

ac and the dc sides. This structure will be referred to as powerfiltered energy control. This structure offers extra flexibility as compared to the previous one, thanks to this LPF. In this case, from (27) it can be seen that $P_{\mathrm{dc}}(s)$ dynamics will depend on the time constant of the filter $\tau_{g 2}$ and the dc gain $k_{g 2}$. With a small $\tau_{g 2}$, the dynamics will be similar to the coupled case. On the contrary, a high $\tau_{g 2}$ would lead to a slower propagation of the disturbance to the dc side, and therefore to an increase of the internal energy. Gain $k_{g 2}$ will be set to 1 from now on, although this value is potentially tunable.

$$
\begin{gathered}
P_{\mathrm{dc}}(s)=\frac{k_{g 2} P_{\mathrm{ac}}(s)}{\left(\tau_{\mathrm{sum}} s+1\right)\left(\tau_{g 2} s+1\right)}+\frac{G_{c}(s)}{\tau_{\mathrm{sum}} s+1} \\
s W_{t}(s)=\left(\frac{k_{g 2} P_{\mathrm{ac}}(s)}{\left(\tau_{\mathrm{sum}} s+1\right)\left(\tau_{g 2} s+1\right)}-P_{\mathrm{ac}}(s)\right)+\frac{G_{c}(s)}{\tau_{\mathrm{sum}} s+1}
\end{gathered}
$$

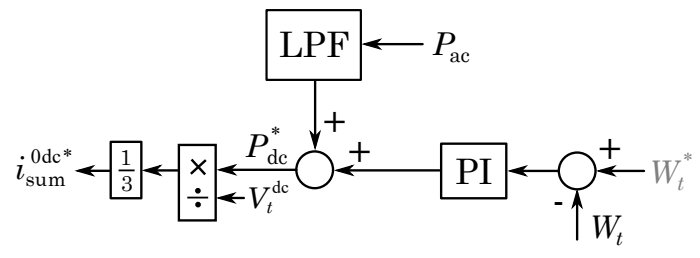

Fig. 9. Power-filtered energy control approach.

\section{Decoupled control approach}

As reported in other studies [34], [42], the energy controller may not include a power feed-forward. In that case, the control effort completely relies on the PI energy controller. In other words, the resulting dynamics of the internal energy and the dc side will completely depend on the PI controller parameters. The more aggressive the controller is, the less the energy will deviate after a disturbance, and the higher the coupling between the ac and the dc sides. This structure will be referred to as decoupled energy control. Then, as in the coupled case, the only tunable part is the PI controller. From (29) it is observed that $P_{\mathrm{dc}}(s)$ does not depend on $P_{\mathrm{ac}}(s)$. Then, the only propagation mechanism relies on the internal energy, i.e., on the PI controller. Looking at (30) it is also noticeable that the energy is directly affected by $P_{\mathrm{ac}}(s)$. Then, it is the job of the PI controller to reject the disturbance, and the resulting $P_{\mathrm{dc}}(s)$ dynamics will depend on that.

$$
\begin{gathered}
P_{\mathrm{dc}}(s)=\frac{G_{c}(s)}{\tau_{\mathrm{sum}} s+1} \\
s W_{t}(s)=-P_{\mathrm{ac}}(s)+\frac{G_{c}(s)}{\tau_{\mathrm{sum}} s+1}
\end{gathered}
$$




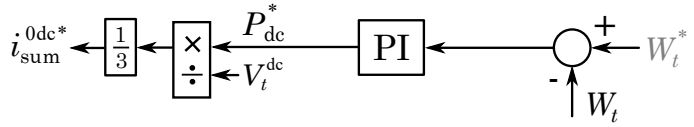

Fig. 10. Decoupled energy control approach.

\section{Dynamic energy reference control approach}

Based on the previous control strategies, the modification of the energy reference signal $\left(W_{t}^{*}\right)$ is proposed and explored as a new alternative. The energy reference will no longer be a constant value but will be changed dynamically, depending on the ac and dc transient power imbalance. Therefore, when there is a power injection from the ac side to the MMC that increases the energy, the energy reference will be decreased automatically. On the contrary, in case of a load connection that decreases the internal energy, the control will increase the energy reference. We define the electrostatic constant of the MMC $\left(H_{c}\right)$, corresponding to the ratio between internal MMC rated energy and the rated power $\left(3 C_{\mathrm{eq}}\left(V_{N}^{\mathrm{dc}}\right)^{2} / P_{N}\right)$, and the parameter $k_{g 4}$ adjusts the rate of change of the energy. This structure will be referred to as dynamic energy reference control. This structure also offers more flexibility as the power-filtered case, with an extra tunable parameter. The corresponding $P_{\mathrm{dc}}$ and $W_{t}$ equations are shown in (31)-(32). In this case, the expressions are more complex, being difficult to make an analytical judgement. Instead, different practical cases will be shown along the next sections.

$$
\begin{aligned}
P_{\mathrm{dc}}(s)= & -\frac{2 H_{c} k_{g}\left(k_{p}+\frac{k_{i}}{s}\right)}{\left(\tau_{\text {sum }} s+1\right)} P_{\mathrm{ac}}(s) \\
& +\left(\frac{2 H_{c} k_{g}\left(k_{p}+\frac{k_{i}}{s}\right)}{\left(\tau_{\mathrm{sum}} s+1\right)}+1\right) G_{c}(s) \\
s W_{t}(s)= & -\left(\frac{2 H_{c} k_{g}\left(k_{p}+\frac{k_{i}}{s}\right)}{\left(\tau_{\text {sum }} s+1\right)}+1\right) P_{\mathrm{ac}}(s) \\
& +\left(\frac{2 H_{c} k_{g}\left(k_{p}+\frac{k_{i}}{s}\right)}{\left(\tau_{\text {sum }} s+1\right)}+1\right) G_{c}(s)
\end{aligned}
$$

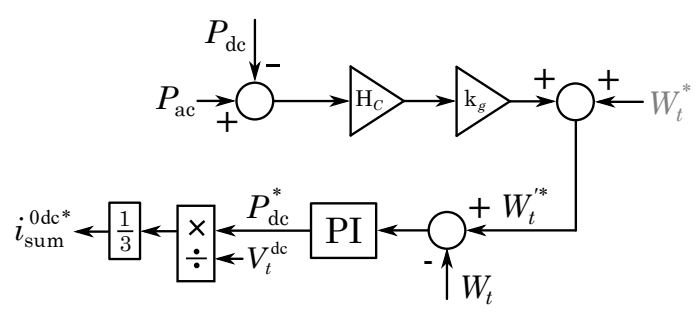

Fig. 11. Dynamic energy reference energy control approach.

\section{E. Summary of the control structures}

A simple model based on (22)-(32) is used to show the transient effect of the ac power disturbance in the dc power and the internal energy of the MMC, summarizing the previous energy control structures (Fig. 12). The initial control parameters are shown in Table I. The ac power positive direction corresponds to the grid-forming supplying a load. It is shown that the coupled approach rapidly propagates the disturbance to the $\mathrm{dc}$ side, as fast as the circulating current control allows for. In the other approaches, the energy fluctuation is much more evident. As an initial example, the parameters $\tau_{g 2}$ and $k_{g 4}$ are changed to show the flexibility that these structures offer. It is noticed that the power-filtered structure is a generic approach that contains the coupled and the decoupled structures as particular cases (corresponding, respectively, to $\tau_{g 2}=0$ and $\tau_{g 2}=\infty$ ). In Section V, the dynamic behavior and capabilities of each structure will be further explored.

TABLE I

CONTROL PARAMETERS RELATED TO THE ENERGY

\begin{tabular}{lccc}
\hline Parameter & Symbol & Value & Units \\
\hline Total energy, proportional & $k_{p}$ & 126 & $\mathrm{~W} / \mathrm{J}$ \\
Total energy, integral & $k_{i}$ & 3948 & $\mathrm{~W} /(\mathrm{Js})$ \\
LPF time constant (pow.-filt.) & $\tau_{g 2}$ & 50 & $\mathrm{~ms}$ \\
LPF dc gain (dyn. ref.) & $k_{g 4}$ & 0.05 & - \\
MMC electrostatic constant & $H_{c}$ & 49.2 & $\mathrm{~ms}$ \\
\hline
\end{tabular}

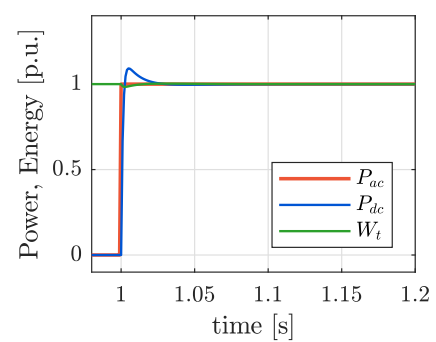

(a) Coupled

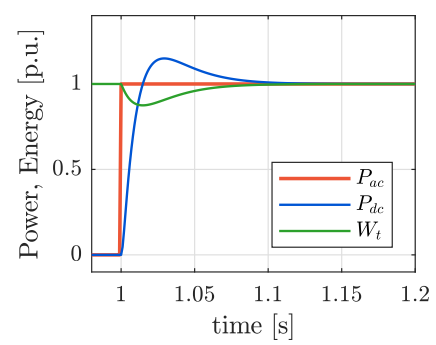

(c) Decoupled

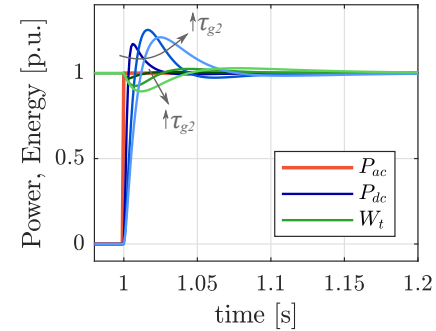

(b) Power-filtered

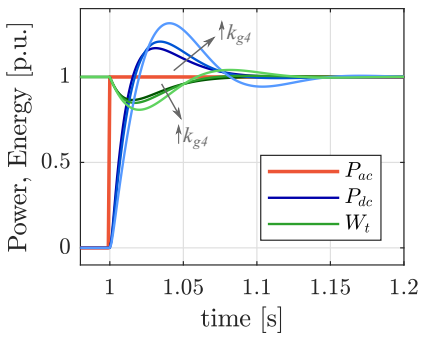

(d) Dynamic $W_{t}^{*}$
Fig. 12. Time-domain plots under a $P_{\mathrm{ac}} 1$ p.u. load step disturbance -Preliminary simplified model (22)-(32)

\section{CASE STUDY}

After presenting the different control structures, a comprehensive analysis evaluating the advantages and drawbacks of each one is addressed in this section using case studies. First of all, an initial case of a single grid-forming MMC is used, for the sake of simplicity in the analysis. Time-domain simulations are carried out using the full non-linear model, consisting of an Average Arm Model (AAM) for the MMC, using the full control scheme from Fig. 4, with saturations in the current references and in the modulation indexes. Then, an eigenvalue analysis using the complete linear model from Fig. 6 is performed in order to identify potential instabilities related to the proposed energy control structures. After, a straightforward tuning method is presented that allows for a simple way to empirically establish a trade-off between the MMC magnitudes 
performance (i.e., energy and dc power). Following up on the results obtained, an optimal tuning methodology is used, in order to unveil the full dynamic capabilities of each structure, tuning all the control parameters at once. Finally, a case scenario considering two grid-forming MMCs operating in parallel is considered, showing the results of the suggested control schemes in a bigger and more realistic scenario.

The ac side grid-forming control structure and its parameters are adopted from previous studies, and it is not within the scope of this paper to address that part of the control system. In the present study, the frequency droop scheme, which is necessary to achieve active power sharing in a multiple gridforming setup, is also implemented in the case of a single gridforming MMC for verification purposes. On the other hand, voltage droop scheme, which is optionally included in some cases for reactive power sharing purposes, is not included for simplicity. System and control parameters related to the ac side are shown in Tables II and III.

TABLE II

MMC AND AC GRID PARAMETERS [8], [19]

\begin{tabular}{lccc}
\hline Parameter & Symbol & Value & Units \\
\hline Rated (base) active power & $P_{N}$ & 500 & $\mathrm{MW}$ \\
Rated (base) AC-side voltage & $U_{N}$ & 320 & $\mathrm{kV}$ \\
Rated (base) DC-side voltage & $V_{N}^{\mathrm{dc}}$ & \pm 320 & $\mathrm{kV}$ \\
Coupling impedance & $R_{s}+\mathrm{j} L_{s}$ & $0.01+\mathrm{j} 0.2$ & $\mathrm{pu}$ \\
Arm reactor impedance & $R_{a}+\mathrm{j} L_{a}$ & $0.01+\mathrm{j} 0.2$ & $\mathrm{pu}$ \\
Converter submodules per arm & $N_{\mathrm{arm}}$ & 400 & - \\
Average submodule voltage & $V_{\mathrm{SM}}$ & 1.6 & $\mathrm{kV}$ \\
Submodule capacitance & $C_{\mathrm{SM}}$ & 8 & $\mathrm{mF}$ \\
AC cable resistance & $r_{c}$ & 0.032 & $\Omega / \mathrm{km}$ \\
AC cable inductance & $l_{c}$ & 0.4 & $\mathrm{mH} / \mathrm{km}$ \\
AC cable capacitance & $c_{c}$ & 0.17 & $\mu \mathrm{F} / \mathrm{km}$ \\
Cable length & $d$ & 25 & $\mathrm{~km}$ \\
\hline
\end{tabular}

TABLE III

CONTROL PARAMETERS RELATED TO THE AC SIDE

\begin{tabular}{lccc}
\hline Parameter & Symbol & Value & Units \\
\hline AC voltage, proportional & $k_{p-u}$ & 0.025 & $\mathrm{~A} / \mathrm{V}$ \\
AC voltage, integral & $k_{i-u}$ & 1 & $\mathrm{~A} /(\mathrm{Vs})$ \\
Frequency droop & $k_{f}$ & $10^{-9}$ & $\mathrm{rad} / \mathrm{W}$ \\
Voltage droop & $k_{u}$ & 0 & - \\
Time constant freq. droop filter & $\tau_{f}$ & 40 & $\mathrm{~ms}$ \\
Time constant AC current control & $\tau_{c c}$ & 1 & $\mathrm{~ms}$ \\
Time constant dc current control & $\tau_{\text {sum }}$ & 1 & $\mathrm{~ms}$ \\
\hline
\end{tabular}

A constant dc voltage source is used to model the dc side of the MMC, assuming that the HVDC grid is robust and stable. The constant PQ node consists of controllable current sources, as mentioned before, including a PLL that tracks the angle in order to inject the corresponding $q d$ current components. These assumptions, which simplify the models and the analysis, do not compromise the validity of the results, as they do not have a relevant impact in the energy dynamics and the disturbance propagation from the ac to the dc side.

\section{A. Single grid-forming $M M C$}

A single grid-forming MMC is considered as an initial case scenario, which is depicted in Fig. 13. The parameters related to the energy control loop are summarized in Table I. The energy PI controller parameters are selected for a settling time of around $100 \mathrm{~ms}$, based on the decoupled structure, as will be further detailed later on.

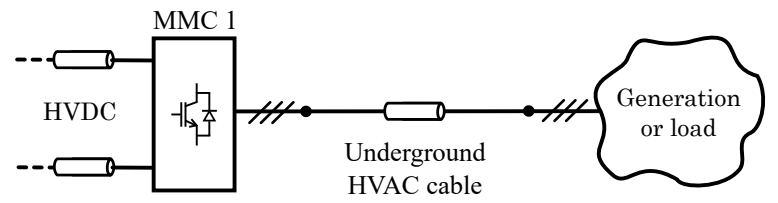

Fig. 13. Initial case study consisting of one grid-forming MMC and a PQ node connected through an ac cable.

In order to highlight the particular capabilities of gridforming as compared to grid-following, the converter is simulated as follows. Initially, a ramping up of the ac voltage (black-start) is performed at $t=0.2 \mathrm{~s}$. Then, the PQ node progressively injects active power to the ac grid at $t=0.4 \mathrm{~s}$, until it reaches 0.5 p.u. (operating point). A 0.25 p.u. step of generated ac active power is performed at $t=1 \mathrm{~s}$ (disturbance), reaching an operating point of 0.75 p.u., which corresponds to approximately 1 p.u. of apparent power. Finally, a sudden disconnection of the PQ node occurs at $t=1.3 \mathrm{~s}$ (large disturbance). Note that the black-start of the voltage and the continuous operation of the converter after a full disconnection in the ac side are not possible in grid-following converters, as they rely on a stable ac grid.

An initial dynamic performance comparison among the different proposed structures, including the case of an equivalent 2L-VSC for completeness, is shown in Fig. 14. On one hand, it can be seen that all the ac side magnitudes are unaffected by the MMC control structure used. In the 2L-VSC case, some similarities are observed in terms of the fundamental oscillation component, although some extra oscillatory components as compared to MMC are shown. On the other hand, regarding the dc magnitudes and the energy, clear dynamic differences can be observed, as discussed next.

In the coupled case, dc current and power responses are similar to the $2 \mathrm{~L}-\mathrm{VSC}$, due to the coupling created by the feed-forward signal. When a low-pass filter is applied to that signal (power-filtered approach), the coupling is reduced, and it is further diminished by eliminating the feed-forward, which corresponds to the decoupled approach. Regarding the dynamic energy reference scheme, the internal energy overshoot is partly compensated, due to the energy reference set point modification during the disturbance. After this initial evaluation, the stability and the performance capabilities of the different structures will be explored in the next subsections.

\section{B. Identification of potential instabilities related to the pro- posed energy control structures}

For a given initial tuning of the energy PI controller, the power-filtered and dynamic energy reference structures offer extra tunable parameters. An eigenvalue analysis of the linear model (Fig. 6) using these two approaches is performed in Figs. 15 and 16, varying these tunable parameters in order to identify potential instabilities: the time constant of the filter $\left(\tau_{g 2}\right)$ in the power-filtered approach, and the dc gain $\left(k_{g 4}\right)$ in the dynamic energy reference approach. Note that an extra dc 

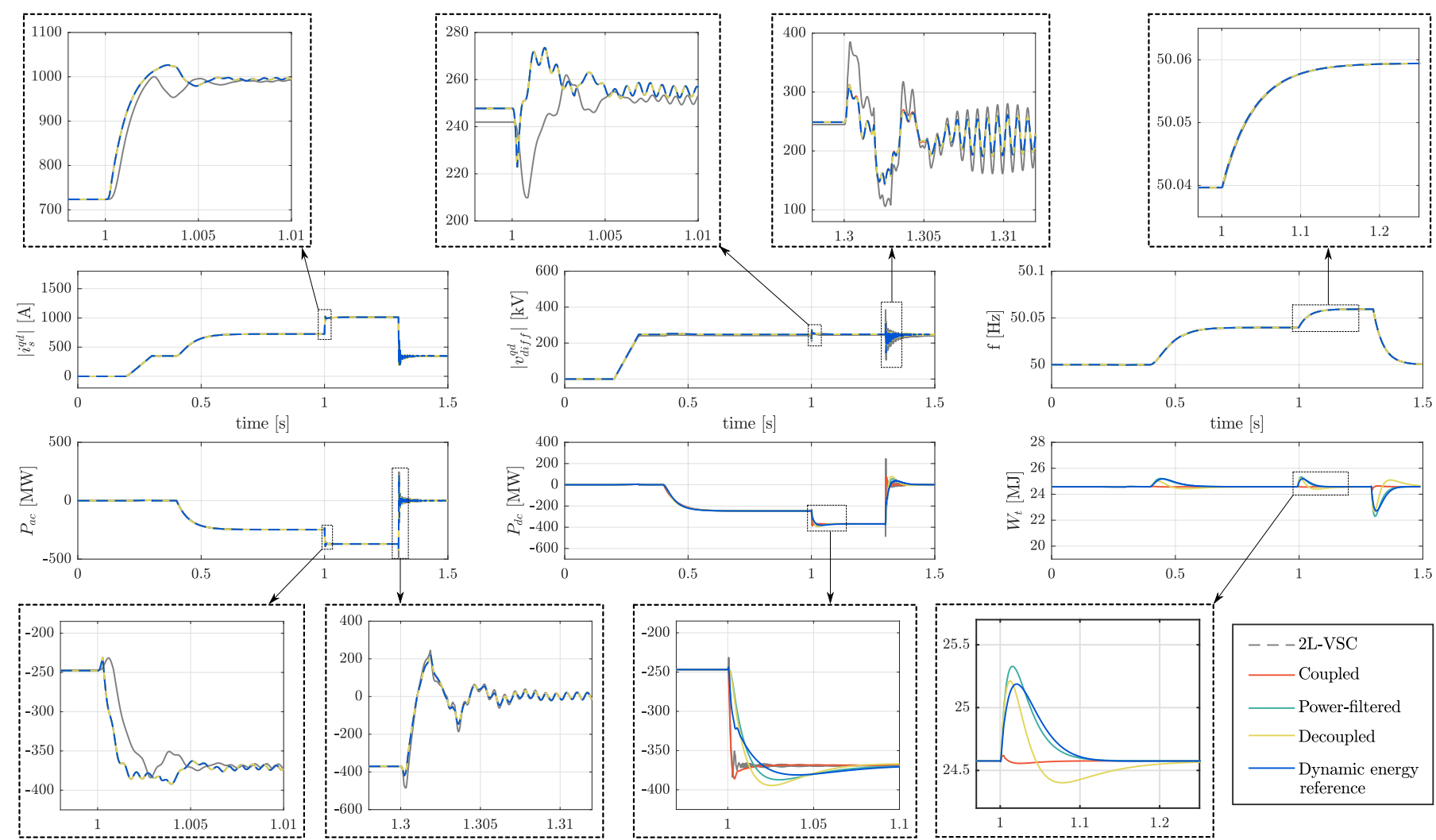

Fig. 14. Time-domain simulation of a single grid-forming MMC: ramping up increase of ac voltage (black-start), slow increase to 0.5 p.u. of generation (operating point), sudden increase of 0.25 p.u. of generation (disturbance), and disconnection of the PQ node (large disturbance) -Dynamic performance of the different energy control structures.

gain in the power-filtered approach would affect the transient response but not the state matrix (i.e., the eigenvalues of the system).

Regarding the power-filtered approach, it is observed that $\tau_{g 2}$ does not cause instability (Fig. 15). One real pole approaches the vertical axis when $\tau_{g 2}$ is increased (a very large value corresponds to the decoupled control approach), but the real part never reaches positive values. On the contrary, when $\tau_{g 2}$ is reduced (zero value corresponds to the coupled control approach) this pole moves away from the vertical axis.

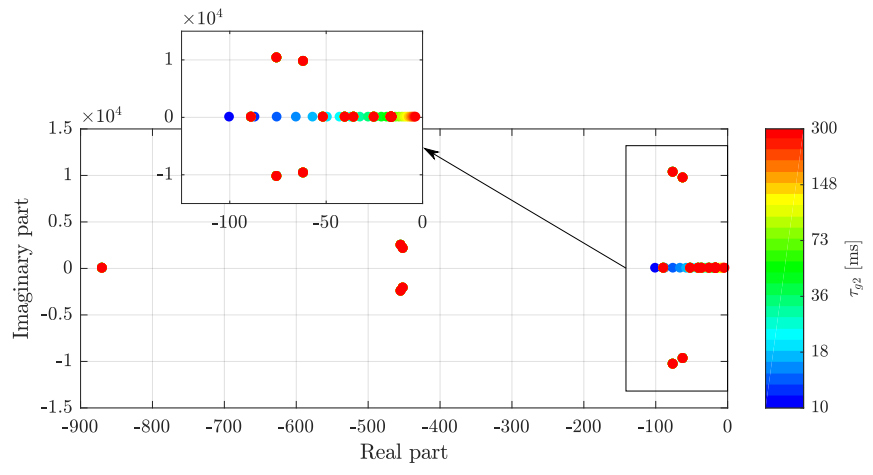

Fig. 15. Eigenvalues of the linear model using the power-filtered approach -Effect of $\tau_{g 2}$

With respect to the dynamic energy reference approach, the effect of $k_{g 4}$ is shown in Fig. 16. A potential instability is detected when this gain is lower than -0.15 (Fig. 16b). If $k_{g 4}$ is positive and not extremely large, it does not compromise the stability (Fig. 16b). However, it will affect the transient performance, as will be explored next.

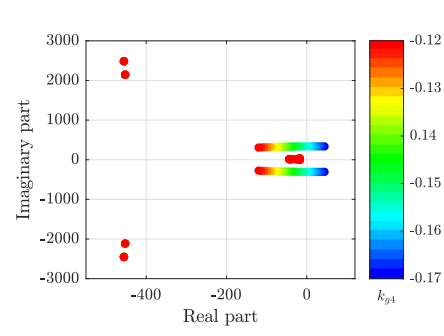

(a) Negative values

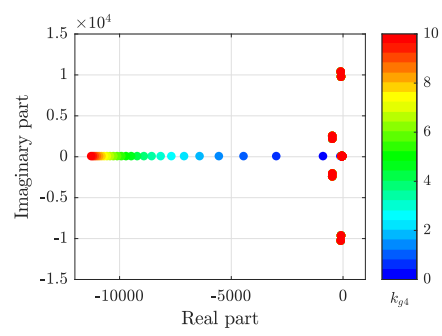

(b) Positive values
Fig. 16. Eigenvalues of the linear model using the dynamic energy reference approach -Effect of $k_{g 4}$.

\section{Empirical tuning}

One of the advantages of the power-filtered and dynamic energy reference structures is that, for a given PI controller, they allow to intuitively tune the control system through a single parameter: $\tau_{g 2}$ and $k_{g 4}$, respectively. This offers some flexibility in terms of establishing a trade-off between the dc power and the energy dynamics.

First, an initial tuning of the energy PI controller has to be established. An initial approximation of the tuning based on reference tracking criteria is adopted, which results in 
a straightforward approach assuming that the system plant is a single MMC internal equivalent capacitor, which is an acceptable assumption if this controller is several times slower than the inner circulating current controller. The decoupled structure is assumed in this case, and the obtained tuning will be used for all the structures. The closed-loop transfer function of this simplified system is

$$
\frac{W_{t}(s)}{W_{t}^{*}(s)}=\frac{s^{2}}{s^{2}+k_{p} s+k_{i}}
$$

and $k_{p}$ and $k_{i}$ can be defined based on a desired secondorder time-domain response, as

$$
k_{p}=2 \xi \omega_{n}, \quad k_{i}=\omega_{n}^{2},
$$

with $\xi=1$ and $\omega_{n}=2 \pi \frac{1}{0.1}$, to achieve a settling time of around $100 \mathrm{~ms}$ (much slower than the current loop) with a critically damped response (i.e., no overshoot). In Fig. 17 it is shown how the power-filtered structure offers a whole range of possibilities through the time constant $\tau_{g 2}$, with the coupled and the decoupled structures as extreme cases (i.e., $\tau_{g 2}=0$ and $\tau_{g 2}=\infty$, respectively). A trade-off solution between the energy deviation and the dc power smoothness can be selected. The higher energy deviation corresponds to the decoupled structure, whereas the sharpest $\mathrm{dc}$ power waveform corresponds to the coupled. Note, however, that for intermediate solutions the dc power overshoot also increases, which might be undesirable.

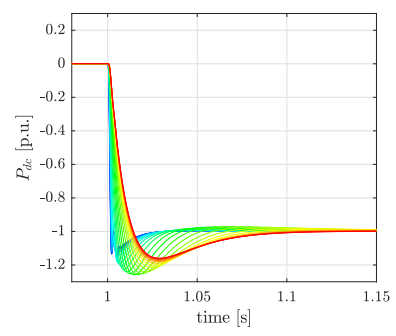

(a) $P_{\mathrm{dc}}(s)$ response

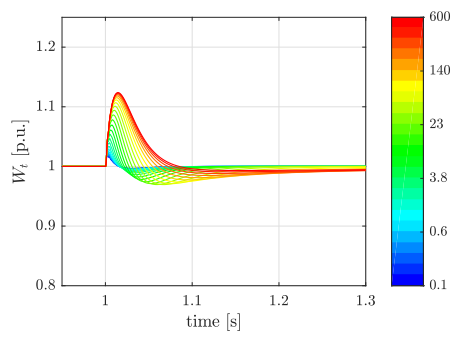

(b) $W_{t}(s)$ response
Fig. 17. Time-domain response under an ac power step disturbance -Powerfiltered structure, $\tau_{g 2}$ sweep.

In Fig. 18, the gain $k_{g 4}$ is swept. It can be seen that increasing $k_{g 4}$ reduces the energy deviation but a high frequency oscillation appears in the dc power. In this case, $k_{g 4}=0.25$ seems to offer a good trade-off between both specifications.

\section{Optimal tuning}

As seen in the previous subsection, the different trade-offs that can be empirically established suggest carrying out an optimal tuning, in order to achieve a specific dynamic requirements or, furthermore, the best possible dynamic response using the different energy control structures. The control system parameters that are potentially tunable are:

- $k_{p}$ : proportional part of the total energy PI.

- $k_{i}$ : integral part of the total energy PI.

- $\tau_{g}$ : time-constant of the low-pass filter (LPF) (only in power-filtered scheme).

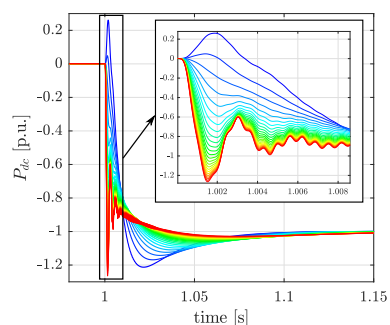

(a) $P_{\mathrm{dc}}(s)$ response

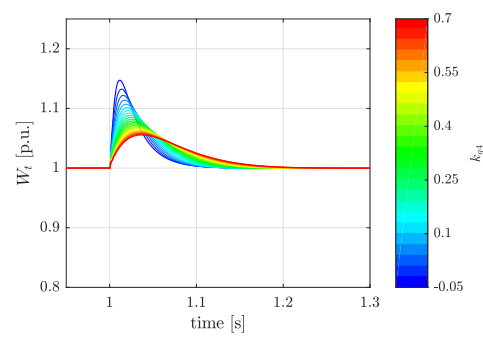

(b) $W_{t}(s)$ response
Fig. 18. Time-domain response under an ac power step disturbance-Dynamic energy reference structure, $k_{g 4}$ sweep.

- $k_{g}$ : dc gain of the low-pass filter (only in dynamic energy reference scheme (note that the optimizer might also add a gain in the power-filtered case.).

To understand the potential capabilities of each presented control structure, in this subsection the tunable parameters of the control system will be tuned using optimization tools. The control objectives are specified as follows. First of all, a $P_{\mathrm{ac}}$ step disturbance of 1 p.u. is considered, so that the system is robust under the worst-case scenario of a sudden disconnection of the generation or the load. Then, we define the transfer functions (35), which are computed using the complete linear model (Fig. 6) and their expressions are not shown for space reasons.

$$
G_{1}(s)=\frac{P_{\mathrm{dc}}(s)}{P_{g}(s)}, \quad G_{2}(s)=\frac{e_{W_{t}}(s)}{P_{g}(s)}
$$

Then, the ideal control objectives are defined as follows:

1) DC power $\left(P_{\mathrm{dc}}\right)$ : a smooth response after a step disturbance, i.e., $0 \%$ overshoot and mitigation of high frequency oscillations. The corresponding gain limit is imposed in the frequency-domain for $G_{1}(s)$, with filtering capability at high frequencies.

2) Internal MMC energy $\left(W_{t}\right)$ : a maximum overshoot of $10 \%$ and relatively fast recovery time. The corresponding gain limit is imposed for $G_{2}(s)$ in the frequencydomain, reducing the gain for low frequencies to achieve integral action (zero error) in the desired settling time.

These specifications prioritize the dc power over the internal energy, in order to use the MMC as a firewall between the ac and the dc sides. Using robust control techniques for a fixed structure controller, the previous objectives can be specified as constraints for an optimization problem that aims to achieve those objectives [43]. In this case, the algorithm is based on the $\mathcal{H}_{\infty}$-norm as described in [44]. Starting with a stable initial tuning, the optimizer iterates on the different tunable parameters and aims to satisfy the previous constraints. In order to understand the limits in terms of dc power and energy, three optimal cases corresponding to three different settling times for the energy are analyzed. In Fig. 19, the results obtained are shown and compared with the initial tuning. Note that these plots are based on the lienar model from Fig. 6. The initial and the optimized tuning values are summarized in Table IV (Appendix B). 


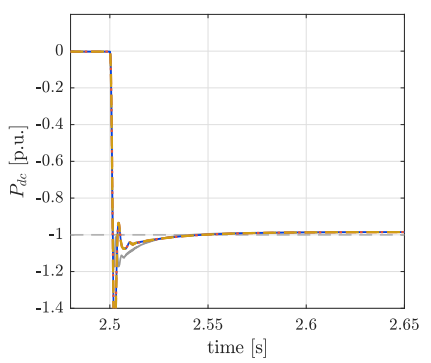

(a) Coupled: $P_{\mathrm{dc}}$ response

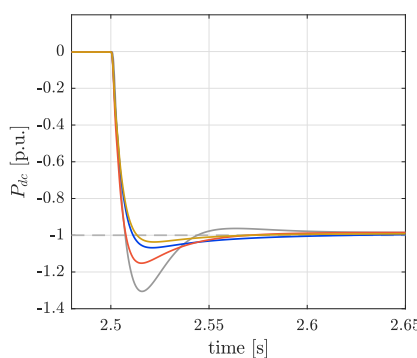

(e) Pow.-filt:: $P_{\mathrm{dc}}$ response

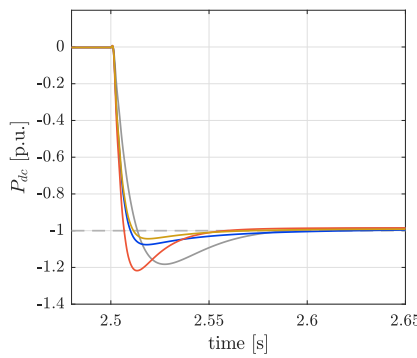

(i) Decoupled: $P_{\mathrm{dc}}$ response

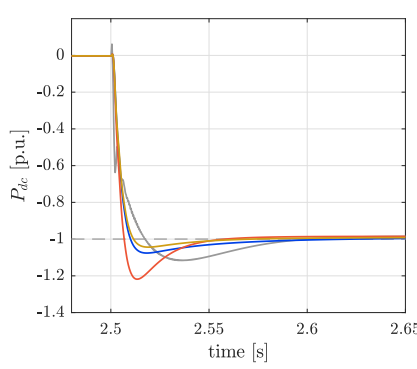

(m) Dyn. $W_{t}^{*}: P_{\mathrm{dc}}$ response

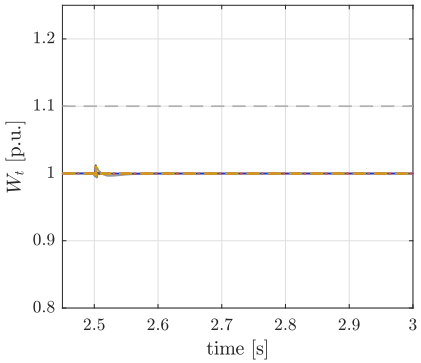

(b) Coupled: $W_{t}$ response

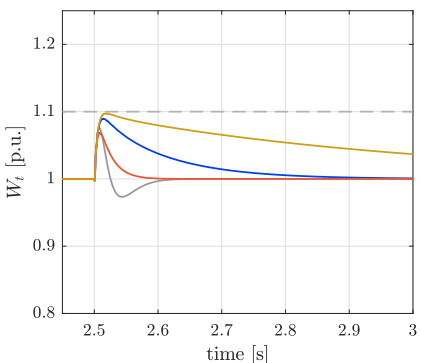

(f) Pow.-filt: $W_{t}$ response

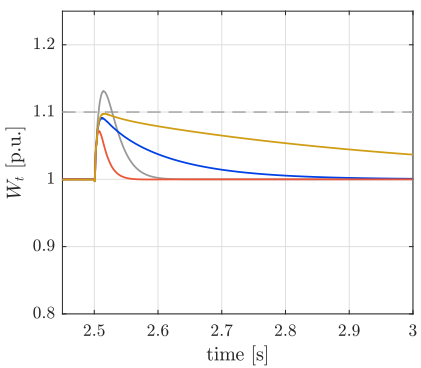

(j) Decoupled: $W_{t}$ response

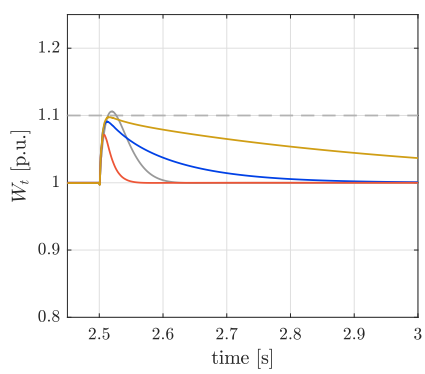

(n) Dyn. $W_{t}^{*}: W_{t}$ response

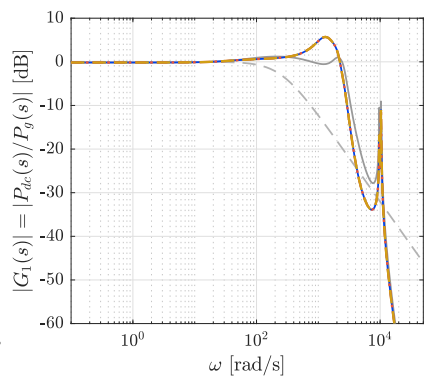

(c) Coupled: $G_{1}(s)$ Bode plot

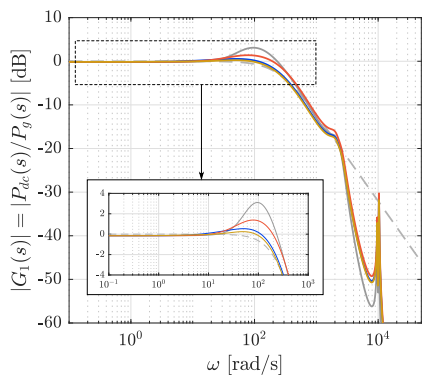

(g) Pow.-filt: $G_{1}(s)$ Bode plot

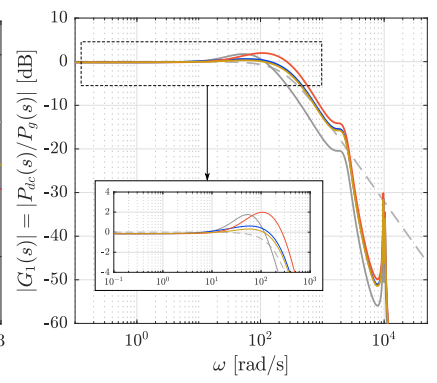

(k) Decoupled: $G_{1}(s)$ Bode plot

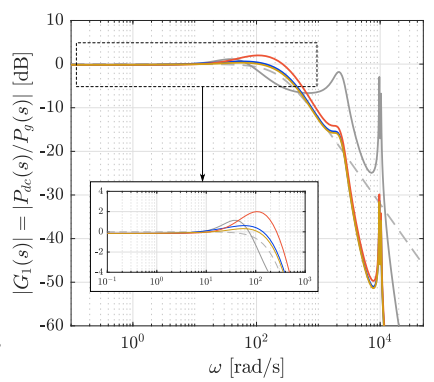

(o) Dyn. $W_{t}^{*}: G_{1}(s)$ Bode plot

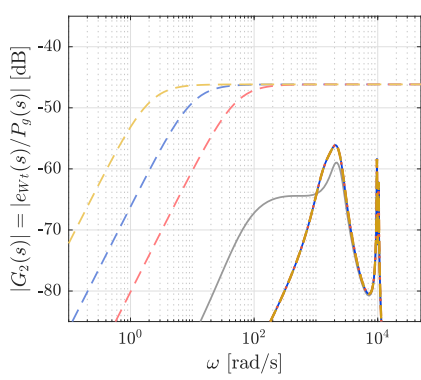

(d) Coupled: $G_{2}(s)$ Bode plot

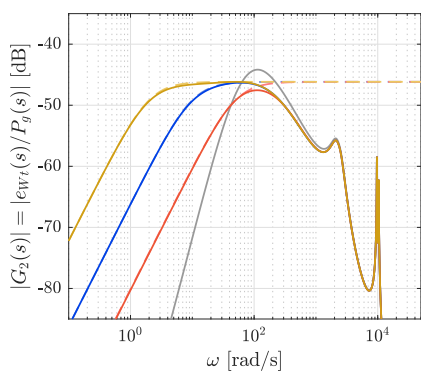

(h) Pow.-filt:: $G_{2}(s)$ Bode plot

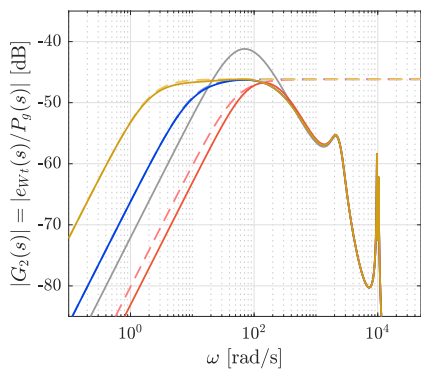

(1) Decoupled: $G_{2}(s)$ Bode plot

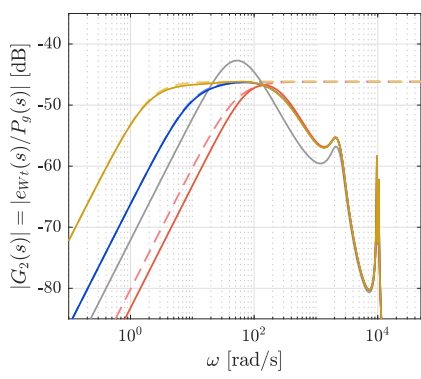

(p) Dyn. $W_{t}^{*}: G_{2}(s)$ Bode plot

- Initial --- Transient limit --- Gain limit $1-$ Opt. $1---$ Gain limit $2-$ Opt. $2---$ Gain limit $3-$ Opt. 3

Fig. 19. Step responses and Bode plots of the design objectives for the different energy control structures, for the initial and different optimal tunings.

First of all, it is shown that the coupled structure is not capable of cancelling the feed-forward effect, and therefore it is not possible to obtain a feasible optimal tuning. This highlights the fact that this structure is not suited to properly enhance the degree of freedom offered by the energy of the MMC. It is shown in Fig. 19c that the optimizer is uncapable of pushing the Bode plot below the limit. The energy deviation that this structure offers is very low, as the ac power feedforward mostly bypasses the buffering capability of the MMC.
Regarding the other control alternatives, it is noticeable that despite all of them show different dynamics using the initial tuning, the optimal solution tends to be equivalent for the three options. This reveals that, at least for the sort of transient requirements specified, neither the power-filtered nor the dynamic energy reference structures offer a clear advantage when an optimal tuning is computed, meaning that a single PI controller (i.e., decoupled structure) can be seen as the simplest solution to obtain the best possible dynamic response. 
To better understand and support this statement, the optimal tuning has been performed under three different constraints, namely Opt. 1, Opt. 2 and Opt. 3. In each case, the constraint related to the settling time of the energy (i.e., the cut-off frequency in Figs. 19d,h,l,p) is changed. It can be seen that the optimizer tends to push the Bode plots to the limits in most cases. This unveils that the reduction of the dc power overshoot comes at the expense of a longer settling time of the internal energy. Going to the limit, an overshoot of $0 \%$ corresponds to an infinite settling time, i.e., droop control of the internal energy. A dc power overshoot between $3-20 \%$ (Figs. 19e,i,m) and an energy settling time of $0.15-1$ seconds (Figs. 19f,j,n) are obtained in the different optimal cases.

This highlights that neither the power-filtered nor the $d y$ namic energy reference structures are able to overcome this limitation with their extra parameters nor to outperform the decoupled alternative, concluding that a single PI controller is sufficient. On one hand, in the power-filtered approach, the gain $k_{g 2}$ is small in the three cases, and the time constant $\tau_{g 2}$ is also very small $(0.07 \mathrm{~ms}$ in all cases $)$, meaning that the filter has almost no effect. On the other hand, in the dynamic energy reference approach, the gain $k_{g 4}$ tends to be almost zero, and the PI parameters are very similar to those of the decoupled structure, meaning that, with an optimal tuning with such requirements, this structure is practically the same as the decoupled one (see Table IV in Appendix B).

\section{E. Multiple grid-forming MMCs}

In more realistic power system applications, more than one converter might be required to operate in grid-forming mode. Then, the ac side control must ensure that the active and reactive power flows are properly shared, which is classically carried out through frequency and voltage droop strategies (Fig. 4). A case scenario with two grid-forming MMCs (Fig. 20) is presented in this final subsection for completeness, where each MMC is using a different energy control structure. This case study represents an offshore wind farm cluster with grid-forming converters exporting power to onshore through two HVDC links.

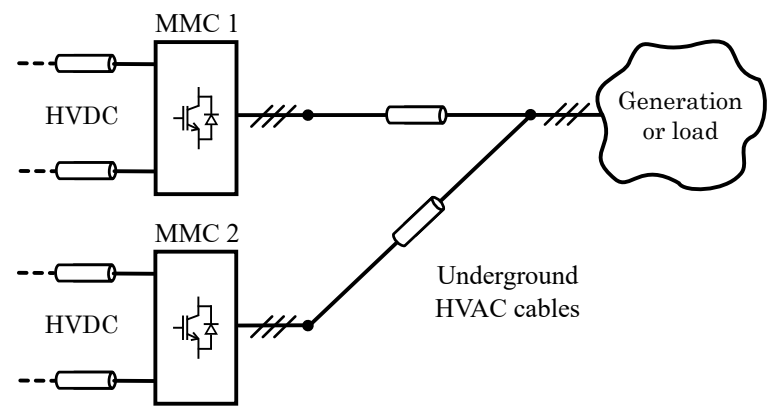

Fig. 20. Case study consisting of two grid-forming MMCs and a PQ node connected through ac cables.

The system initial operating point is set to 1 p.u. of ac power flowing from the ac side to the converters (each converter is absorbing 0.5 p.u. thanks to the frequency droop control equal power sharing feature). A step of generation coming from the PQ node to the grid-forming MMCs of 0.5 p.u.
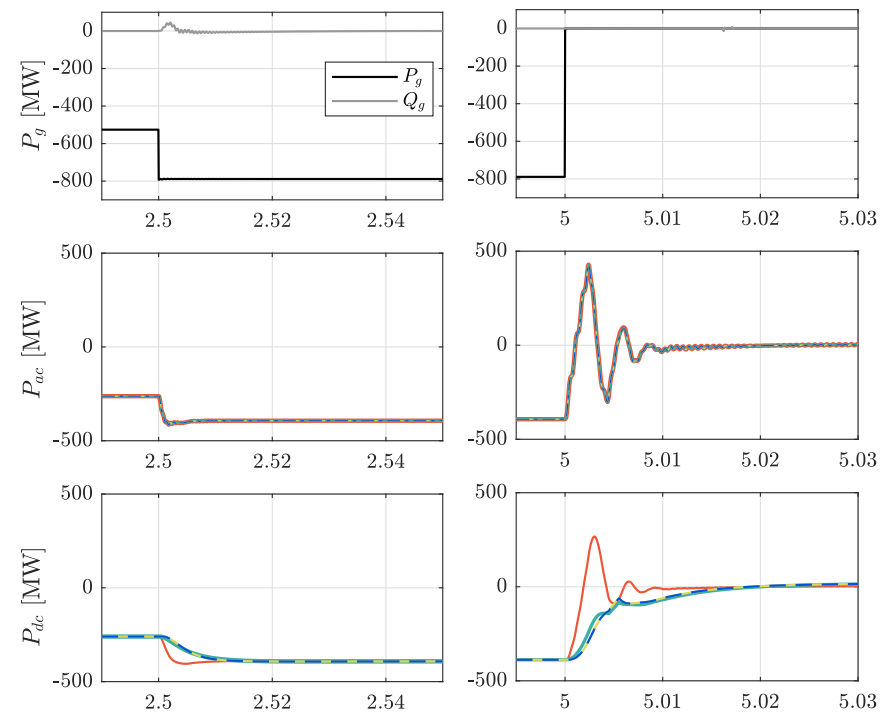

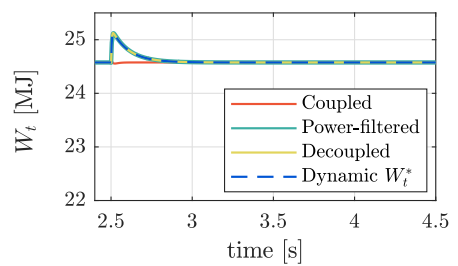

(a) Positive step of 0.5 p.u.

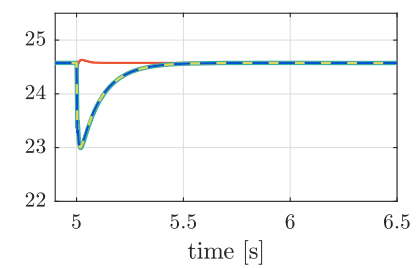

(b) Sudden line disconnection
Fig. 21. Time-domain simulation (multiple grid-forming MMCs) - Dynamic performance using coupled (MMC 1) and decoupled (MMC 2) control structures under positive and negative steps of generation.

is performed at $t=2.5 \mathrm{~s}$, and a full disconnection of the wind farm is performed at $t=5 \mathrm{~s}$. The coupled structure with the initial tuning is used for the first MMC, whereas for the second MMC the power-filtered, deoupled and dynamic energy reference structures using the optimal tuning (Opt. 2) are implemented and compared (Fig. 21).

Despite the same ac powers being seen in both MMCs, the performance in terms of dc power and internal energy of each MMC is independent from each other, and it completely depends on the energy control structure used. In the case of the coupled approach (MMC 1), even though the dc power shows a significantly attenuated response with respect to the ac power disturbance, the response is not smooth and some undesired overshoot is observed, specially when the line disconnects (Fig. 21b). On the other hand, in MMC 2 the disturbance is completely blocked thanks to the capabilities offered by the power-filtered, deoupled and dynamic energy reference structures under the optimal tuning, which allows for achieving a smooth dc power with barely no overshoot and without compromising the internal energy limits. Also, it is shown that these three structures exhibit practically the same transient characteristics. Some non-linearities are observed in the dc power in Fig. 21b, which may be caused by the current saturation of the ac current control and the saturation of the modulation indexes during the transient after the disconnection of the line. Nevertheless, there is no noticeable overshoot in the dc power, and the energy deviates only up to around $6 \%$. 


\section{CONCLUSION}

The buffering feature inherent to the MMC topology can be used to prevent ac side power disturbances to propagate to the $\mathrm{dc}$ side when operating in grid-forming mode if an adequate control of its internal energy is performed. As HVDC grids are likely to experience a growth in the upcoming years, this mitigation feature becomes crucial in order to preserve the correct operation of the whole dc system, transiently isolating sudden power changes that may occur in the different ac connection points, specially in the case of large and complex meshed HVDC grids. In this paper, different control structures for the energy control of the MMC have been explored. On one hand, this degree of freedom related to the energy of the MMC can be effectively exploited with the powerfiltered, decoupled and dynamic reference structures. The power-filtered and dynamic reference options allow for an intuitive and straightforward control tuning through a single parameter. Furthermore, it was demonstrated by optimally tuning the control parameters of these structures with highly restrictive requirements that these three structures exhibit equivalent transient performance features. On the other hand, the coupled structure is not recommended, as it does not effectively mitigate the propagation of ac side disturbances to the dc side, bypassing to a greater or lesser extent the energy buffer of the MMC. Ultimately, it can be concluded that the grid-forming MMC under the aforementioned optimal tuning exhibits a high dynamic performance under severe ac power disturbances, with a small deviation of the internal energy and a smooth dc power response with a very small overshoot.

\section{APPENDIX A \\ REFERENCE CHANGE TRANSFORMATIONS}

The following matrices are used for the transformations between the PQ node and the grid-forming MMC $q d$ reference frames as in (20)-(21). Note that the $q d$ components of the operating point values $\left(x_{0}\right)$ are expressed in the initial reference, i.e., grid-forming MMC reference (common frame) in the case of (36), and PQ node reference (auxiliary frame) in (37). If more auxiliary $q d$ frames were present in the system (i.e., due to additional grid-forming MMCs or PQ nodes), $x_{0}$ in (37) would be expressed in its corresponding frame.

$$
\begin{aligned}
\mathbf{T}_{\mathbf{c}}^{\mathbf{q d}} & =\left[\begin{array}{ccc}
\cos \left(\theta_{0}\right) & -\sin \left(\theta_{0}\right) & -\sin \left(\theta_{0}\right) x_{0}^{q}-\cos \left(\theta_{0}\right) x_{0}^{d} \\
\sin \left(\theta_{0}\right) & \cos \left(\theta_{0}\right) & \cos \left(\theta_{0}\right) x_{0}^{q}-\sin \left(\theta_{0}\right) x_{0}^{d}
\end{array}\right] \\
\mathbf{T}_{\mathbf{c}}^{\mathbf{q d}{ }^{-1}} & =\left[\begin{array}{ccc}
\cos \left(\theta_{0}\right) & \sin \left(\theta_{0}\right) & -\sin \left(\theta_{0}\right) x_{0}^{q c}+\cos \left(\theta_{0}\right) x_{0}^{d c} \\
-\sin \left(\theta_{0}\right) & \cos \left(\theta_{0}\right) & -\cos \left(\theta_{0}\right) x_{0}^{q c}-\sin \left(\theta_{0}\right) x_{0}^{d c}
\end{array}\right]
\end{aligned}
$$

\section{APPENDIX B \\ INITIAL AND OPTIMAL TUNING}

\section{REFERENCES}

[1] P. Fragkos, N. Tasios, L. Paroussos, P. Capros, and S. Tsani, "Energy system impacts and policy implications of the European Intended Nationally Determined Contribution and low-carbon pathway to 2050," Energy Policy, vol. 100, pp. 216-226, 2017.
TABLE IV

\begin{tabular}{|c|c|c|c|c|c|c|}
\hline & & Initial & Opt. 1 & Opt. 2 & Opt. 3 & Units \\
\hline \multirow{2}{*}{ Coupled } & $k_{p}$ & 126 & 1854 & 1854 & 1854 & $\mathrm{~W} / \mathrm{J}$ \\
\hline & $k_{i}$ & 3948 & 17 & 126 & 4 & $\mathrm{~W} /(\mathrm{Js})$ \\
\hline \multirow{4}{*}{ Power-filtered } & $k_{p}$ & 126 & 187 & 224 & 180 & $\mathrm{~W} / \mathrm{J}$ \\
\hline & $k_{i}$ & 3948 & 1708 & 8434 & 344 & $\mathrm{~W} /(\mathrm{Js})$ \\
\hline & $\tau_{g 2}$ & 10 & 0.07 & 0.07 & 0.07 & $\mathrm{~ms}$ \\
\hline & $k_{g 2}$ & 1 & 0.143 & 0.165 & 0.137 & - \\
\hline \multirow{2}{*}{ Decoupled } & $k_{p}$ & 126 & 219 & 252 & 211 & $\mathrm{~W} / \mathrm{J}$ \\
\hline & $k_{i}$ & 3948 & 1997 & 14186 & 400 & $\mathrm{~W} /(\mathrm{Js})$ \\
\hline \multirow{3}{*}{ Dynamic $W_{t}^{*}$} & $k_{p}$ & 126 & 219 & 252 & 211 & $\mathrm{~W} / \mathrm{J}$ \\
\hline & $k_{i}$ & 3948 & 1998 & 14215 & 400 & $\mathrm{~W} /(\mathrm{Js})$ \\
\hline & $k_{g 4}$ & 0.1 & 0.0003 & 0.0005 & 0.0003 & - \\
\hline
\end{tabular}

INITIAL AND OPTIMAL TUNING PARAMETERS

[2] N. Pogaku and M. Prodanovic, "Inverter-based microgrids: Small-signal modelling and testing," in 3rd Int. Conf. Power Electron. Mach. Drives, 2006, pp. 499-504.

[3] S. D'Arco and J. A. Suul, "Virtual synchronous machines - Classification of implementations and analysis of equivalence to droop controllers for microgrids," 2013 IEEE Grenoble Conf. PowerTech, POWERTECH 2013, 2013

[4] Z. Li, S. Member, C. Zang, P. Zeng, and H. Yu, "Control of A GridForming Inverter Based on Sliding Mode and Mixed $\mathrm{H}_{2} / \mathrm{H}_{\infty}$ Control," IEEE Trans. Ind. Electron., vol. 64, no. 5, pp. 3862-3872, 2017.

[5] A. Navarro-Rodríguez, P. García, J. M. Cano, and M. Sumner, "Limits, Stability and Disturbance Rejection Analysis of Voltage Control Loop Strategies for Grid Forming Converters in DC and AC Microgrids with High Penetration of Constant Power Loads," in 19th Eur. Conf. Power Electron. Appl. EPE-ECCE Eur., 2017, pp. 1-10.

[6] L. Antonio De Souza Ribeiro, F. D. Freijedo, F. De Bosio, M. Soares Lima, J. M. Guerrero, and M. Pastorelli, "Full Discrete Modeling, Controller Design, and Sensitivity Analysis for High-Performance GridForming Converters in Islanded Microgrids," IEEE Trans. Ind. Appl., vol. 54, no. 6, pp. 6267-6278, 2018.

[7] L. Zeni, B. Hesselbaek, P. E. Sorensen, A. D. Hansen, and P. C. Kjaer, "Control of VSC-HVDC in offshore AC islands with wind power plants: Comparison of two alternatives," IEEE Eindhoven PowerTech, 2015.

[8] M. Raza, E. Prieto-Araujo, and O. Gomis-Bellmunt, "Small-Signal Stability Analysis of Offshore AC Network Having Multiple VSCHVDC Systems," IEEE Trans. Power Deliv., vol. 33, no. 2, pp. 830-839, 2018.

[9] G. Denis, T. Prevost, M.-S. Debry, F. Xavier, X. Guillaud, and A. Menze, "The Migrate project: the challenges of operating a transmission grid with only inverter-based generation. A grid-forming control improvement with transient current-limiting control," IET Renew. Power Gener. vol. 12 , no. 5, pp. 523-529, 2018.

[10] J. Rocabert, A. Luna, F. Blaabjerg, and P. Rodríguez, "Control of power converters in AC microgrids," IEEE Trans. Power Electron., vol. 27, no. 11, pp. 4734-4749, 2012.

[11] S. D'Arco and J. A. Suul, "Equivalence of virtual synchronous machines and frequency-droops for converter-based Microgrids," IEEE Trans. Smart Grid, vol. 5, no. 1, pp. 394-395, 2014.

[12] S. I. Nanou and S. A. Papathanassiou, "Evaluation of Control Schemes for Island VSC-HVDC Links Operating in Parallel with AC Interconnectors," in IEEE Manchester PowerTech, 2017.

[13] Y. Jing, R. Li, L. Xu, and Y. Wang, "Enhanced AC voltage and frequency control on offshore MMC station for wind farm," J. Eng., vol. 2017, no. 13, pp. 1264-1268, 2017.

[14] F. Mahr and J. Jaeger, "Advanced Grid-Forming Control of HVDC Systems for Reliable Grid Restoration," in IEEE Power Energy Soc. Gen. Meet. IEEE, 2018, pp. 1-5.

[15] H. Saad, S. Dennetière, and P. Rault, "AC Fault dynamic studies of islanded grid including HVDC links operating in VF-control," in 15th IET Int. Conf. AC DC Power Transm., 2019, pp. 1-10.

[16] S. Henninger, M. Schroeder, and J. Jaeger, "Grid-Forming Droop Control of a Modular Multilevel Converter in Laboratory," in IEEE Power Energy Soc. Gen. Meet. IEEE, 2018, pp. 1-5.

[17] S. D'Arco, G. Guidi, and J. A. Suul, "Operation of a Modular Multilevel Converter Controlled as a Virtual Synchronous Machine," 2018 Int. Power Electron. Conf. IPEC-Niigata - ECCE Asia 2018, pp. 782-789, 2018.

[18] Z. Lv and Q. C. Zhong, "Control of modular multilevel converters as 
virtual synchronous machines," IEEE Power Energy Soc. Gen. Meet., pp. 1-5, 2018.

[19] E. Prieto-Araujo, A. Junyent-Ferré, C. Collados-Rodríguez, G. ClarianaColet, and O. Gomis-Bellmunt, "Control design of Modular Multilevel Converters in normal and AC fault conditions for HVDC grids," Electr. Power Syst. Res., vol. 152, pp. 424-437, 2017.

[20] Q. Tu, Z. Xu, and L. Xu, "Reduced Switching-frequency modulation and circulating current suppression for modular multilevel converters," IEEE Trans. Power Deliv., vol. 26, no. 3, pp. 2009-2017, 2011.

[21] A. Antonopoulos, L. Angquist, and H.-P. Nee, "On dynamics and voltage control of the Modular Multilevel Converter," in 13th Eur. Conf. Power Electron. Appl., 2009

[22] G. Bergna, J. A. Suul, E. Berne, P. Egrot, P. Lefranc, J. C. Vannier, and M. Molinas, "Mitigating DC-side power oscillations and negative sequence load currents in Modular Multilevel Converters under unbalanced faults-First approach using resonant PI," IECON Proc. (Industrial Electron. Conf., pp. 537-542, 2012.

[23] G. Bergna-Díaz, J. A. Suul, E. Berne, J. C. Vannier, and M. Molinas, "Optimal Shaping of the MMC Circulating Currents for Preventing ACSide Power Oscillations from Propagating into HVdc Grids," IEEE J. Emerg. Sel. Top. Power Electron., vol. 7, no. 2, pp. 1015-1030, 2019.

[24] G. Bergna, J. A. Suul, E. Berne, J. C. Vannier, and M. Molinas, "MMC circulating current reference calculation in $\mathrm{ABC}$ frame by means of Lagrange Multipliers for ensuring constant DC power under unbalanced grid conditions," 2014 16th Eur. Conf. Power Electron. Appl. EPE-ECCE Eur. 2014, pp. 1-10, 2014.

[25] K. Shinoda, A. Benchaib, J. Dai, and X. Guillaud, "Virtual Capacitor Control: Mitigation of DC Voltage Fluctuations in MMC-Based HVdc Systems," IEEE Trans. Power Deliv., vol. 33, no. 1, pp. 455-465, 2018.

[26] G. Bergna, J. A. Suul, and S. D'Arco, "Impact on small-signal dynamics of using circulating currents instead of AC-currents to control the DC voltage in MMC HVDC terminals," in IEEE Energy Convers. Congr. Expo., 2016, pp. 1-8.

[27] E. Sánchez-Sánchez, E. Prieto-Araujo, A. Junyent-Ferré, and O. GomisBellmunt, "Analysis of MMC Energy-based Control Structures for VSCHVDC Links," IEEE J. Emerg. Sel. Top. Power Electron., vol. 6, no. 3 , pp. 1065-1076, 2018.

[28] L. Harnefors, A. Antonopoulos, S. Norrga, L. Angquist, and H. P. Nee, "Dynamic analysis of modular multilevel converters," IEEE Trans. Ind. Electron., vol. 60, no. 7, pp. 2526-2537, 2013.

[29] H. Saad, X. Guillaud, J. Mahseredjian, S. Dennetière, and S. Nguefeu, "MMC Capacitor Voltage Decoupling and Balancing Controls," IEEE Trans. Power Deliv., vol. 30, no. 2, pp. 704-712, 2015.

[30] S. Rohner, J. Weber, and S. Bernet, "Continuous model of modular multilevel converter with experimental verification," in IEEE Energy Convers. Congr. Expo. Energy Convers. Innov. a Clean Energy Futur. ECCE. IEEE, 2011, pp. 4021-4028.

[31] P. C. Krause, O. Wasynczuk, and S. D. Sudhoff, Analysis of Electric Machinery and Drive Systems, 2002.

[32] G. Bergna-Diaz, J. Freytes, X. Guillaud, S. D'Arco, and J. A. Suul, "Generalized Voltage-based State-Space Modelling of Modular Multilevel Converters with Constant Equilibrium in Steady-State," IEEE J. Emerg. Sel. Top. Power Electron., vol. 6, no. 2, pp. 707-725, 2018

[33] G. Bergna-Diaz, J. A. Suul, and S. D'Arco, "Energy-Based State-Space Representation of Modular Multilevel Converters with a Constant Equilibrium Point in Steady-State Operation," IEEE Trans. Power Electron., vol. 33, no. 6, pp. 4832-4851, 2018.

[34] G. Bergna Diaz, J. A. Suul, and S. D'Arco, "Small-signal state-space modeling of modular multilevel converters for system stability analysis," in IEEE Energy Convers. Congr. Expo. ECCE, 2015, pp. 5822-5829.

[35] S. K. Chung, "A phase tracking system for three phase utility interface inverters," IEEE Trans. Power Electron., vol. 15, no. 3, pp. 431-438, 2000.

[36] H. Akagi, E. H. Watanabe, and M. Aredes, Instantaneous power theory and Applications to power conditioning. Wiley, 2007.

[37] L. Zhang, "Modeling and Control of VSC-HVDC Links Connected to Weak AC Systems," Ph.D. dissertation, Royal Institute of Technology, 2010.

[38] J. Freytes, S. Akkari, J. Dai, F. Gruson, P. Rault, and X. Guillaud, "Small-signal state-space modeling of an HVDC link with modular multilevel converters," in IEEE 17th Work. Control Model. Power Electron. COMPEL, 2016.

[39] K. Shinoda, J. Freytes, A. Benchaib, J. Dai, H. Saad, and X. Guillaud, "Energy Difference Controllers for MMC without DC Current Perturbations," in 2nd Int. Conf. HVDC, Shanghai, China, 2016.

[40] J. Freytes, G. Bergna, J. A. Suul, S. D’Arco, F. Gruson, F. Colas, H. Saad, and X. Guillaud, "Improving Small-Signal Stability of an MMC with CCSC by Control of the Internally Stored Energy," IEEE Trans. Power Deliv., vol. 33, no. 1, pp. 429-439, 2018.

[41] J. Freytes, P. Rault, F. Gruson, F. Colas, and X. Guillaud, "Dynamic impact of MMC controllers on DC voltage droop controlled MTDC grids," 2016 18th Eur. Conf. Power Electron. Appl. EPE 2016 ECCE Eur., pp. 1-10, 2016.

[42] J. Beerten, G. B. Diaz, S. D' Arco, and J. A. Suul, "Comparison of small-signal dynamics in MMC and two-level VSC HVDC transmission schemes," IEEE Int. Energy Conf. ENERGYCON, 2016.

[43] P. Apkarian, P. Gahinet, and C. Buhr, "Multi-model, multi-objective tuning of fixed-structure controllers," Eur. Control Conf. ECC, pp. 856861, 2014.

[44] P. Apkarian and D. Noll, "Nonsmooth $H_{\infty}$ synthesis," IEEE Trans. Automat. Contr., vol. 51, no. 1, pp. 71-86, 2006. 